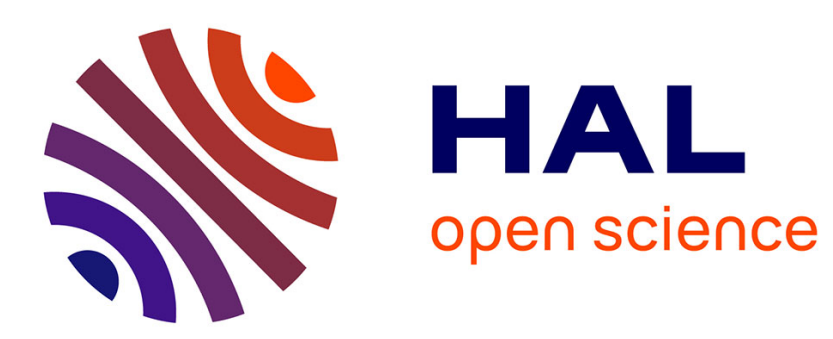

\title{
A Type-Theoretic Foundation of Delimited Continuations
}

\author{
Zena Ariola, Hugo Herbelin, Amr Sabry
}

\section{To cite this version:}

Zena Ariola, Hugo Herbelin, Amr Sabry. A Type-Theoretic Foundation of Delimited Continuations. Higher-Order and Symbolic Computation, 2007. inria-00177326

\section{HAL Id: inria-00177326 \\ https://hal.inria.fr/inria-00177326}

Submitted on 7 Oct 2007

HAL is a multi-disciplinary open access archive for the deposit and dissemination of scientific research documents, whether they are published or not. The documents may come from teaching and research institutions in France or abroad, or from public or private research centers.
L'archive ouverte pluridisciplinaire HAL, est destinée au dépôt et à la diffusion de documents scientifiques de niveau recherche, publiés ou non, émanant des établissements d'enseignement et de recherche français ou étrangers, des laboratoires publics ou privés. 


\title{
A Type-Theoretic Foundation of Delimited Continuations ${ }^{\dagger}$
}

\author{
Zena M. Ariola \\ University of Oregon \\ Hugo Herbelin \\ INRIA-Futurs \\ Amr Sabry ${ }^{\S}$ \\ Indiana University
}

\begin{abstract}
There is a correspondence between classical logic and programming language calculi with first-class continuations. With the addition of control delimiters, the continuations become composable and the calculi become more expressive. We present a fine-grained analysis of control delimiters and formalise that their addition corresponds to the addition of a single dynamically-scoped variable modelling the special top-level continuation. From a type perspective, the dynamically-scoped variable requires effect annotations. In the presence of control, the dynamically-scoped variable can be interpreted in a purely functional way by applying a store-passing style. At the type level, the effect annotations are mapped within standard classical logic extended with the dual of implication, namely subtraction. A continuation-passing-style transformation of lambda-calculus with control and subtraction is defined. Combining the translations provides a decomposition of standard CPS transformations for delimited continuations. Incidentally, we also give a direct normalisation proof of the simply-typed lambda-calculus with control and subtraction.
\end{abstract}

Keywords: callcc, monad, prompt, reset, shift, subcontinuation, subtraction

\section{Introduction}

Programming practice suggests that control operators add expressive power to purely functional languages. For example, control operators permit the implementation of backtracking (Haynes, 1986), coroutines (Haynes et al., 1986), and lightweight processes (Wand, 1999), which go beyond pure functional programming. Of course, any complete program which uses these abstractions can be globally transformed using the continuation-passing style (CPS) transformation to a purely

\footnotetext{
$\dagger$ Extended version of the conference article "A Type-Theoretic Foundation of Continuations and Prompts." (Ariola et al., 2004)

¥ Supported by National Science Foundation grant number CCR-0204389

$\S$ Supported by National Science Foundation grant number CCR-0204389, by a Visiting Researcher position at Microsoft Research, Cambridge, U.K., and by a Visiting Professor position at the University of Genova, Italy.
}

(C) 2007 Kluwer Academic Publishers. Printed in the Netherlands. 
functional program, but this misses the point. As Felleisen (1990) formalises and proves, the additional expressiveness of control operators comes from the fact that no local transformation of program fragments using control operators is possible.

There is another, simpler, way to formalise the additional expressive power of control operators that is based on the Curry-Howard isomorphism (Howard, 1980). The pure $\lambda$-calculus corresponds to (minimal) intuitionistic logic; extending it with the control operator $\mathcal{C}$ (Felleisen, 1988) makes it expressive enough to computationally interpret classical logic proofs (Griffin, 1990). Felleisen (1990) also showed that the control operator callcc is less expressive than $\mathcal{C}$. The logical counterpart is that callcc is enough to computationally express proofs of minimal classical logic where it is possible to prove Peirce's law but not double negation elimination, whereas $\mathcal{C}$ corresponds to (non-minimal) classical logic which proves double negation elimination (see Ariola and Herbelin, 2003).

So far, only a small family of control operators have been shown to be connected to proof constructions in logic, and the situation for the other control operators is much less understood. In particular, the addition of control delimiters (Felleisen, 1988) gives rise to delimited continuations which are more expressive than their undelimited counterparts (Sitaram and Felleisen, 1990b). Even for the well-studied operators for delimited continuations shift and reset (Danvy and Filinski, 1990), it is not clear how to formalise this additional expressiveness for at least three reasons:

1. Several type systems and type-and-effect systems have been proposed for these operators (Danvy and Filinski, 1989; Murthy, 1992; Kameyama, 2000; Kameyama, 2001), and it is not apparent whether one of these systems is the "right" one even when moving to the worlds of CPS or monads (Wadler, 1994). Furthermore, for the type-and-effect systems, there are several possible interpretations of the effect annotations, which should be completely eliminated anyway to get a correspondence with a standard logic.

2. Although the semantics of many control operators (including shift and reset (Kameyama and Hasegawa, 2003)) can be given using local axioms, a few properties of these systems, when seen as rewriting systems, have been investigated. Especially, many of the systems are not confluent, and under none of the type-and-effect systems is a property such as strong normalisation known to be ensured.

3. Delimited continuations can simulate a large number of other computational effects like state and exceptions (Filinski, 1994). This 
seems to indicate that the understanding of the expressive power of such control operators must also include an understanding of the expressive power of other effects. Riecke and Thielecke (1999) and Thielecke $(2000,2001)$ have formalised the expressive power of various combinations of continuations, exceptions and state but their results are not expressed using standard type systems and logics.

We show that the additional expressiveness of control delimiters can be characterised in terms of a dynamically-scoped continuation variable, and that in the presence of dynamic binding, continuations can model other effects like state and exceptions.

Environment-passing transformation is the standard way to interpret dynamic binding in a functional way. In the presence of control, it turns out that a store-passing transformation is needed. This is because the dynamically-scoped continuation variable can be passed back to the contexts surrounding the binding locations.

At the level of types, if a continuation in the store is assigned the type $\neg T$, then a store-passing transformation translates functional types of the form $A \rightarrow B$ into types of the form $A \wedge \neg T \rightarrow B \wedge \neg T$. Interestingly, we have the following equivalences that hold classically but not intuitionistically:

$$
A \wedge \neg T \rightarrow B \quad \text { iff } \quad A \rightarrow B \vee T \quad \text { iff } \quad A \wedge \neg T \rightarrow B \wedge \neg T
$$

This informally complies with the fact that in the presence of control delimiters, all of the following particular cases are definable: functions which refer to a dynamic variable of type $\neg T$ without relying on control, functions which throw an exception of type $T$, functions which manipulate a state variable of type $\neg T$.

Felleisen's approach of the theory of control (Felleisen, 1988) relies on a reification of evaluation contexts into continuations. Contrastingly, our approach, inspired by Ariola and Herbelin (2003), itself inspired by Parigot (1992), directly deals with contexts (or more precisely with elementary pieces of context).

Passing around a store which is a context requires to be able to manipulate the pair of a term and of a context. From this perspective, the connective of logical subtraction $A-T$ (Rauszer, 1974; Crolard, 2001; Crolard, 2004), which is classically equivalent to $A \wedge \neg T$, naturally arises. This connective will be used to interpret the effect annotations.

The next section reviews some background material on abortive continuations. We present Felleisen and Hieb (1992) revised theory of control and the $\lambda_{\mathcal{C}_{\mathrm{p}}}$-calculus, an extended syntactic variant of call-byvalue $\lambda \mu$-calculus (Parigot, 1992; Ong and Stewart, 1997) from previous 


$$
\begin{array}{ll}
x, a, v, f, c, k \in \text { Vars } & \\
M, N \in \text { Terms } & :=x|\lambda x . M| M N \mid \mathcal{C} M \\
V \in \text { Values } & :=x \mid \lambda x . M
\end{array}
$$

Figure 1. Syntax of $\lambda_{\mathcal{C}}$

work by the first two authors (Ariola and Herbelin, 2003). Section 3 explains the semantics of delimited continuations by generalising the $\lambda_{\mathcal{C} \text { tp }}$-calculus to include just one dynamically-scoped variable. Section 4 investigates various ways to extend the type system of $\lambda_{\mathcal{C} \text { tp }}$ to accommodate the dynamically-scoped variable. We present three systems (closely related to existing type-and-effect systems) and reason about their properties. Sections 5 and 6 explain how to interpret the effect annotations in a standard logic. The first focuses on motivating the dual connective of implication, namely subtraction, and formally defining $\lambda_{\mathcal{C}}^{-}$, a calculus with control operators and subtraction, but no dynamic binding. The second focuses on using the subtractive type for explaining the action on the effect annotations of a store-passing style transformation when what is stored is an evaluation context. Section 7 develops a CPS transformation for $\lambda_{\mathcal{c}}^{-}$that ultimately shows that known CPS transformations for delimited continuations are decomposable into a store-passing style transformation into $\lambda_{\mathcal{C}}^{-}$followed by a standard CPS transformation into the pure $\lambda$-calculus. The first transformation takes care of the control delimiter; the second transformation takes care of the control operator. Section 8 concludes with a discussion of some of the other control operators. Also discussed is the Curry-Howard problem

for control delimiters. Finally, Appendix A gives the details of the proof of strong normalisation for simply-typed $\lambda_{\mathcal{c}}^{-}$.

\section{Abortive Continuations and Classical Logic}

We review the semantics of abortive continuations and their connection to classical logic. We start with Felleisen and Hieb (1992) revised theory of control.

\subsection{The $\lambda_{\mathcal{C}}$-Calculus: Felleisend and Hieb Revised theory OF CONTROL}

Figure 1 introduces the syntax of a call-by-value calculus extended with the unary operator $\mathcal{C}$. Variables and lambda-abstractions are called values. There is no distinction between continuations and variables, and a continuation does not need to be applied. The reduction semantics is 


\begin{tabular}{lll|}
$\beta_{v}:$ & $(\lambda x . M) V$ & $\rightarrow M[V / x]$ \\
$\mathcal{C}_{L}:$ & $(\mathcal{C} M) N$ & $\rightarrow \mathcal{C}(\lambda c . M(\lambda f \cdot \mathcal{A}(c(f N))))$ \\
$\mathcal{C}_{R}:$ & $V(\mathcal{C} M)$ & $\rightarrow \mathcal{C}(\lambda c . M(\lambda x . \mathcal{A}(c(V x))))$ \\
$\mathcal{C}_{\text {idem }}: \mathcal{C}(\lambda c . \mathcal{C} M)$ & $\rightarrow \mathcal{C}(\lambda c . M(\lambda x . \mathcal{A} x))$ \\
$\mathcal{C}_{\text {top }}:$ & $\mathcal{C} M$ & $\rightarrow \mathcal{C}(\lambda c . M(\lambda x . \mathcal{A}(c x)))$ \\
\hline
\end{tabular}

Figure 2. Reduction rules of call-by-value $\lambda_{\mathcal{C}}$ (Felleisen and Hieb)

$$
\begin{aligned}
x, a, v, f \in \text { Vars } & \\
k \in \text { KVars } & \\
\text { KConsts } & =\{\text { tp }\} \\
M, N \in \text { Terms } & :=x|\lambda x . M| M N \mid \mathcal{C}(\lambda k . J) \\
V \in \text { Values } & ::=x \mid \lambda x . M \\
J \in \text { Jumps } & :=k M \mid \text { tp } M
\end{aligned}
$$

Figure 3. Syntax of $\lambda_{\mathcal{C} \text { tp }}$

given in Figure 2. Our previous work (2003) introduced an alternative theory of control called $\lambda_{\mathcal{C} \text { tp }}$ (For a thorough comparison of the two systems see (Ariola and Herbelin, 2007)).

\subsection{The $\lambda_{\mathcal{C} \text { tp }}$-Calculus: Syntax and Dynamic Semantics}

The syntax of the call-by-value $\lambda_{\mathcal{C} \text { tp }}$-calculus is in Figure 3 . It is a callby-value variant of Parigot's $\lambda \mu$-calculus (1992) where $\mu$ is written $\mathcal{C}$. Ariola and Herbelin (2003) showed that a top-level continuation constant has to be added to the $\lambda \mu$-calculus to recover the expressiveness of the control calculus of Felleisen and Hieb (1992). The resulting calculus has the following properties:

- every use of the control operator $\mathcal{C}$ must be syntactically applied to a procedure which receives the captured continuation. For example, whereas $\lambda y . \mathcal{C} y$ is a legal $\lambda_{\mathcal{C}}$ term, it is not a legal $\lambda_{\mathcal{C} \text { tp }}$ term;

- variables bound to continuations are distinct from other variables and can only occur in application position. For example, whereas $\mathcal{C}(\lambda k . k)$ or $\mathcal{C}(\lambda k . \lambda x . k)$ are legal $\lambda_{\mathcal{C}}$ terms, they are not legal $\lambda_{\mathcal{C} \text { tp }}$ terms;

- applications of continuations are called jumps and must occur exactly when a continuation is also captured. For example, whereas $\mathcal{C}(\lambda k .(k x) y)$ is a legal $\lambda_{\mathcal{C}}$ term, it is not a legal $\lambda_{\mathcal{C} \text { tp }}$ term;

- there is a continuation constant tp which denotes the top-level continuation. 


$$
\begin{aligned}
(x)^{\circ} & =x \\
(\lambda x . M)^{\circ} & =\lambda x \cdot M^{\circ} \\
(M N)^{\circ} & =M^{\circ} N^{\circ} \\
(\mathcal{C} M)^{\circ} & =\mathcal{C}\left(\lambda k \cdot \operatorname{tp}\left(M^{\circ}(\lambda x \cdot \text { Th } k x)\right)\right)
\end{aligned}
$$

Figure 4. Translation of $\lambda_{\mathcal{C}}$ in $\lambda_{\mathcal{C} \text { tp }}$

$$
\begin{array}{ll}
\beta_{v}: & (\lambda x . M) V \rightarrow M[V / x] \\
\mathcal{C}_{L}: & \mathcal{C}(\lambda k . J) N \rightarrow \mathcal{C}(\lambda k . J[k(\square N) / k]) \\
\mathcal{C}_{R}: & V \mathcal{C}(\lambda k . J) \rightarrow \mathcal{C}(\lambda k . J[k(V \square) / k]) \\
\mathcal{C}_{\text {elim }}: & \mathcal{C}(\lambda k . k M) \rightarrow M \quad \text { where } k \notin F V(M) \\
& \\
\mathcal{C}_{\text {idem }}: & \operatorname{tp} \mathcal{C}(\lambda k . J) \rightarrow J[\operatorname{tp} \square / k] \\
\mathcal{C}_{\text {idem }}: & k^{\prime} \mathcal{C}(\lambda k . J) \rightarrow J\left[k^{\prime} \square / k\right]
\end{array}
$$

Figure 5. Reductions of call-by-value $\lambda_{\mathcal{C} \text { tp }}$

We sometimes use the following convenient abbreviations:

$$
\begin{aligned}
\mathcal{A} M & \triangleq \mathcal{C}\left(\lambda_{-} . \text {tp } M\right) \\
\text { Th } k M & \triangleq \mathcal{C}\left(\lambda_{-} . k M\right)
\end{aligned}
$$

(Abbrev. 1)

(Abbrev. 2)

In both abbreviations, the variable _ is an anonymous continuation variable that does not occur in the body of the abstraction. Thus, the first abbreviation corresponds to jumping to the top-level continuation. The second abbreviation corresponds to jumping to a previously-captured continuation $k$.

The translation of $\lambda_{\mathcal{C}}$ terms into the $\lambda_{\mathcal{C} \text { tp }}$-calculus is given in Figure 4. For example, $\lambda y . \mathcal{C} y$ becomes $\lambda y \cdot \mathcal{C}(\lambda k$.tp $(y(\lambda x . \mathcal{T h} k x)))$. Notice how the captured continuation is given a name $k$; the implicit jump to the top-level associated with the continuation capture is made explicit; and the implicit aborting of the context when $k$ is applied is also made explicit.

The semantics is given by the local reduction rules in Figure 5. The first four reduction rules are similar to the ones given by Felleisen and Hieb (1992) for $\lambda_{\mathcal{C}}$ but with important differences discussed below:

- Like the original calculus, the rules $\mathcal{C}_{L}$ and $\mathcal{C}_{R}$ allow one to lift the control operation step-by-step until it reaches a point where it can no longer be lifted. When the control operator reaches a jump to the top-level (rule $\mathcal{C}_{\text {idem }}$ ), the continuation captured is the trivial continuation modelled by tp.

- Unlike $\lambda_{\mathcal{C}}$, the reduction rules of Figure 5 use structural substitutions for continuations. The general form of structural substitution 
relies on the definition of contexts:

$$
E::=\square|E M| V E,
$$

and on the notation $E[N]$ which stands for the replacement of the hole $\square$ of $E$ with $N$. The structural substitution $M[q E / k]$ (resp. $J[q E / k]$ ) reads as: "replace every jump of the form $k N$ in $M$ (resp. $J$ ) by the jump $(q E[N])$ (and recursively in $N$ )." The substitutions $M[\operatorname{tp} E / k]$ and $J[\operatorname{tp} E / k]$ are defined similarly. For example,

$$
\operatorname{tp} \mathcal{C}\left(\lambda k . k\left(M \mathcal{C}\left(\lambda k^{\prime} . k N\right)\right)\right) \rightarrow \operatorname{tp}\left(M \mathcal{C}\left(\lambda k^{\prime} . \operatorname{tp} N\right)\right)
$$

assuming that $k$ does not occur free in $M$ and $N$. What is distinctive about this structural substitution is that it is performed irrespective of whether the jump argument is a value or not. In particular, in the above example, the application of $M$ is clearly not a value and $N$ need not be a value. In contrast, $\lambda_{\mathcal{C}}$ requires the argument to every jump to be reduced to a value before performing the jump.

- Unlike the original presentation, the abortive behaviour of $\mathcal{C}$ is not hard-wired in the reduction rules but in the syntax. Each call $k M$ to a continuation $k$ bound by some $\mathcal{C}$ is necessarily surrounded by some other $\mathcal{C}\left(\lambda k^{\prime} . \square\right)$ making it abortive. Contrastingly, the original reduction rules dynamically insert the context $\mathcal{C}\left(\lambda_{-} . \square\right)$ around each reference to $k$ (an abortive operator which is useless, as shown by the original $\mathcal{C}_{\text {idem }}$, when $k$ is already surrounded by some $\mathcal{C}$ ).

Rule $\mathcal{C}_{\text {idem }}$ is a generalisation of $\mathcal{C}_{\text {idem }}$ which, apart from the notion of structural substitution, is similar to the rule $M(\mathcal{C} N) \rightarrow N(\lambda x .(M x))$ where $M$ has type $\neg A$, proposed by Barbanera and Berardi (1993). In terms of the $\lambda \mu$-calculus, both $\mathcal{C}_{\text {idem }}{ }^{\prime}$ and $\mathcal{C}_{\text {idem }}$ correspond to Parigot's "renaming" rule (Parigot, 1992). The $\mathcal{C}_{\text {elim }}$ rule, which is also used by Hofmann (1995) for completeness reasons, was also proposed by Felleisen and Hieb (1992) since it leads to a better simulation of evaluation. However, unlike our system, Felleisen and Hieb extended reduction theory is non-confluent.

The difference between $\lambda_{\mathcal{C}}$ and $\lambda_{\mathcal{C} \text { tp }}$ is not observable with respect to reduction to values (Ariola and Herbelin, 2007):

PROPOSITION 1. Let $M$ be a closed $\lambda_{\mathcal{C}}$ term:

$$
\mathcal{A} M \rightarrow{ }_{\lambda_{\mathcal{C}}} \mathcal{A} V \text { iff tp } M^{\circ} \rightarrow_{\lambda_{\mathcal{C} t p}} \text { tp } V^{\prime} .
$$




$$
\begin{gathered}
b \in \text { BaseType } \\
A, B, T \in \text { TypeExp }:=b \mid A \rightarrow B \\
\Gamma \in \text { Contexts }::=\cdot|\Gamma, x: A| \Gamma, k: A \rightarrow \Perp \\
\frac{\Gamma, x: A \vdash x: A ; T}{\Gamma} A x \\
\frac{\Gamma, x: A \vdash M: B ; T}{\Gamma \vdash \lambda x . M: A \rightarrow B ; T} \rightarrow_{i} \frac{\Gamma \vdash M: A \rightarrow B ; T \quad \Gamma \vdash M^{\prime}: A ; T}{\Gamma \vdash M M^{\prime}: B ; T} \rightarrow_{e} \\
\frac{\Gamma, k: A \rightarrow \Perp \vdash J: \Perp ; T}{\Gamma \vdash \mathcal{C}(\lambda k . J): A ; T} R A A \\
\frac{\Gamma, k: A \rightarrow \Perp \vdash M: A ; T}{\Gamma, k: A \rightarrow \Perp \vdash k M: \Perp ; T} \rightarrow{ }_{e}^{k} \frac{\Gamma \vdash M: T ; T}{\Gamma \vdash \operatorname{tp} M: \Perp ; T} \rightarrow_{e}^{\mathrm{tp}}
\end{gathered}
$$

Figure 6 . Type system of $\lambda_{\mathcal{C} \text { tp }}$

However, it is significant as the use of structural substitution avoids introducing useless and artificial $\beta$-expansions which are not even $\beta_{v^{-}}$ expansions. In contrast, the $\beta$-expansions in the original calculus force both the evaluation of the argument to the jump in some continuation and then the erasure of this continuation, instead of the equivalent but more natural (and efficient) choice of first erasing the continuation and then evaluating the argument to the jump.

\subsection{The $\lambda_{\text {ctp }}$-Calculus: Static Semantics}

The calculus $\lambda_{\mathcal{c t p}}$ was originally motivated by the Curry-Howard correspondence between classical logic and control operators. The calculus is both a clarification of the connection between the control operator $\mathcal{C}$ and classical logic (Griffin, 1990), and a counterpart to Parigot's $\lambda \mu$ calculus for a formulation of classical logic based on the classical rule Reductio Ad Absurdum (see below).

According to the Curry-Howard correspondence, the natural interpretation of the tp continuation constant is as a constructor for the elimination rule of the "false" connective $\perp$, i.e., as a primitive continuation expecting an argument of type $\perp$. In the current approach, we generalise the use of tp. We assign it the role of the top-level continuation for a top-level that can have any fixed but arbitrary type and not only the type $\perp$ as in the characterisation of (non-minimal) classical logic.

The resulting system of simple types for $\lambda_{\mathcal{C} \text { tp }}$ is given in Figure 6 . The set of base types in $\lambda_{\mathcal{C} \text { tp }}$ could include $\perp$ to represent an empty type or the proposition "false" but that type plays no special role in 
the judgements. The judgements do however refer to a special type $\Perp$ which is used as the type of jumps, i.e., the type of expressions in the syntactic category $J$. The type $\Perp$ is not a base type; it can never occur as the conclusion of any judgement for terms in the syntactic category $M$, but it may occur in the context $\Gamma$ as the return type of a continuation variable. We sometimes abbreviate $T \rightarrow \Perp$ as $\neg \Perp T$.

The judgements are of two forms: $\Gamma \vdash M: A ; T$ for typing terms and $\Gamma \vdash J: \Perp ; T$ for typing jumps. In both cases the type $T$ is the arbitrary but fixed type for the top-level; it acts as a global parameter to the type system (Murthy, 1992). The rule RAA (Reductio Ad Absurdum) is similar to the double-negation rule in Griffin's system (1990) except that it uses the special type $\Perp$ instead of $\perp$. According to the $\rightarrow{ }_{e}^{\text {tp }}$ rule, the special top-level continuation can only be invoked with a term of the distinguished type $T$. According to the $\rightarrow_{e}^{k}$ rule, continuation variables are invoked with a term of the type expected by the context in which they were captured.

It is possible to inject a continuation of type $T \rightarrow \Perp$ into the type of functions $T \rightarrow \perp$ :

$$
\begin{aligned}
& \overline{\Gamma, k: T \rightarrow \Perp, x: T \vdash x: T ; T} \\
& \overline{\Gamma, k: T \rightarrow \Perp, x: T \vdash k x: \Perp ; T} \\
& \frac{\overline{\Gamma, k: T \rightarrow \Perp, x: T \vdash \mathcal{C}\left(\lambda_{\_} \cdot k x\right): \perp ; T}}{\Gamma, k: T \rightarrow \Perp \vdash \lambda x . \mathcal{C}\left(\lambda_{-} . k x\right): T \rightarrow \perp ; T}
\end{aligned}
$$

Thus, it does no harm to informally think of $\Perp$ as $\perp$ remembering that an explicit coercion is required to move from one to the other (the way to coerce $T \rightarrow \perp$ into $T \rightarrow \Perp$ is given in Intermezzo 16). But the special nature of the type $\Perp$ can perhaps be best understood by examining the situation in the isomorphic $\lambda \mu$-calculus extended with tp. In that type system, there is no need for $\Perp$ : the types of continuation variables are maintained on the right-hand side of the sequent and jumps have no

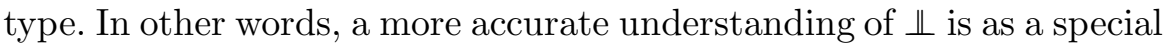
symbol denoting "no type."

Simple experience with the type system reveals that the top-level type sometimes provides helpful additional information about the type of a term. For example:

$$
\vdash \mathcal{A} 5==\text { "Hello" : bool; int }
$$

accurately predicts that the expression returns a bool or jumps to the top-level with an int. However, the top-level type sometimes provides misleading information about the type of a term. For example, in the judgement:

$$
\vdash \lambda x .(x+\mathcal{A} \text { "Hello" }): \text { int } \rightarrow \text { int; string }
$$


the presence of the type string is actually associated with the definition of the function and not its use. This confusion could lead to problems as discussed in Sections 4.1 and 4.2 where we talk about generalisations of the system.

\section{PROPOSITION 2.}

(i) $\lambda_{\mathcal{C} t p}$ is confluent.

(ii) Subject reduction: Given $\lambda_{\mathcal{C} t p}$ terms $M$ and $N$, if $\Gamma \vdash M: A ; T$ and $M \rightarrow N$, then $\Gamma \vdash N: A ; T$.

(ii) Progress: A closed $\lambda_{\mathcal{c t p}}$ jump $J$ is either a jump to the toplevel with a value (i.e. a jump tp $V$ ) or a head-reducible jump (i.e. either of the form tp $E[N]$ with $N$ a redex or tp $\mathcal{C}(\lambda k . J)$ which is a redex).

(ii) Simply typed terms are strongly normalising.

Proof. Confluence is shown in (Ariola and Herbelin, 2007) where it relies on the standard parallel reduction method, using extra tricks from (Baba et al., 2001). Subject reduction and progress are routine verifications. Strong normalisation is somehow standard but it can also be obtained as a consequence of Proposition 17, interpreting tp as a free continuation variable.

\section{Delimited Continuations}

We modify the $\lambda_{\mathcal{C t p}}$-calculus by adding control delimiters. We then show that the delimiter can be eliminated in favour of a single dynamicallyscoped variable.

\subsection{Adding Control Delimiters}

As shown in Figure 7, we extend the syntax of the $\lambda_{\mathcal{C} \text { tp }}$ calculus with a control delimiter \#. The use of \# delimits the continuation captured by $\mathcal{C}$. For example, the occurrence of $\mathcal{C}$ in:

$$
1+(\#(\operatorname{tp}(2+(\mathcal{C}(\lambda k \ldots)))))
$$

only captures the continuation corresponding to the context $(2+\square)$. Similarly, in the following expression, the use of \# delimits the jump:

$$
1+(\#(\text { tp } 2))
$$




$$
\begin{aligned}
x, a, v, f \in \text { Vars } & \\
k \in \text { KVars } & \\
\text { KConsts } & =\{\text { tp }\} \\
M, N \in \text { Terms } & :=x|\lambda x . M| M N|\mathcal{C}(\lambda k . J)| \# J \\
V \in \text { Values } & ::=x \mid \lambda x . M \\
J \in \text { Jumps } & ::=k M \mid \operatorname{tp} M
\end{aligned}
$$

Figure 7 . Syntax of $\lambda_{\mathcal{C} \# \text { tp }}$

$$
\begin{array}{lll}
\beta_{v}: & (\lambda x . M) V & \rightarrow M[V / x] \\
\mathcal{C}_{L}: & \mathcal{C}(\lambda k . J) N \rightarrow \mathcal{C}(\lambda k . J[k(\square N) / k]) \\
\mathcal{C}_{R}: & V(\mathcal{C}(\lambda k . J)) \rightarrow \mathcal{C}(\lambda k . J[k(V \square) / k]) \\
\mathcal{C}_{\text {idem }}: & \operatorname{tp} \mathcal{C}(\lambda k . J) & \rightarrow J[\operatorname{tp} \square / k] \\
\mathcal{C}_{\text {idem }}: & k^{\prime} \mathcal{C}(\lambda k . J) & \rightarrow J\left[k^{\prime} \square / k\right] \\
\mathcal{C}_{\text {elim }}: & \mathcal{C}(\lambda k . k M) & \rightarrow M \quad \text { where } k \notin F V(M) \\
\#_{v}: & \#(\operatorname{tp} V) & \rightarrow V
\end{array}
$$

Figure 8 . Reductions of call-by-value $\lambda_{\mathcal{C} \# \text { tp }}$

The jump to the top-level is blocked by the \#, and the entire expression should evaluate to 3 . This intuition is consistent with the original motivation for introducing control delimiters: they provide an explicit "top-level" for the evaluation of a subexpression (Felleisen, 1988).

The semantics is formalised by adding one new reduction to the reductions of $\lambda_{\mathcal{C} \text { p }}$ as shown in Figure 8 . The reduction $\#_{v}$ formalises that the role of the \# terminates once its subexpression has been simplified to a value, which by definition can no longer perform any control actions.

$\lambda_{\mathcal{C} \text { tp }}$ is sound and complete equivalent with respect to Felleisen's $\lambda_{\mathcal{C} \#}$ calculus 1988, which consists of adding \#-expressions to the syntax of $\lambda_{\mathcal{C}}$. The reduction semantics of $\lambda_{\mathcal{C} \#}$ has the following two rules for the $\#$ :

$$
\begin{array}{ll}
\#_{\mathcal{c}} \#(\mathcal{C} M) & \rightarrow \#(M(\lambda x \cdot \mathcal{A} x)) \\
\#_{v} \# V & \rightarrow V
\end{array}
$$

but omit the $\mathcal{C}_{\text {top }}$ rule. The translation of Figure 4 is extended with the clause:

$$
(\# M)^{\circ}=\#\left(\operatorname{tp} M^{\circ}\right)
$$

The equivalence can then be stated as follows (Ariola and Herbelin, 2007):

PROPOSITION 3. Let $M$ be a closed $\lambda_{\mathcal{C} \#}$ term:

$$
\# M \rightarrow{ }_{\lambda_{\mathcal{C} \#}} V \text { iff \# }\left(\text { tp } M^{\circ}\right) \rightarrow{ }_{\lambda_{\mathcal{C} \# t p}} V^{\prime}
$$


REMARK 4. The presence of the delimiter \# makes it possible to express the operators shift and reset given in the seminal paper of Danvy and Filinski (1989). Filinski (1994) showed that both operators are definable from Felleisen's $\mathcal{C}$ and \# operators. Expressed in $\lambda_{\mathcal{C} \# \text { tp }}$, we get:

$$
\begin{array}{ll}
\mathcal{S}(\lambda q \cdot M) & =\mathcal{C}(\lambda k \cdot \operatorname{tp}(\lambda q \cdot M)(\lambda x . \#(k x)))) \\
\langle M\rangle & =\#(\operatorname{tp} M)
\end{array}
$$

The essence of the above definition is to surround each invocation of a continuation by the delimiter. This however does not mean that each jump in $\lambda_{\mathcal{C} \# \text { tp }}$ must be surrounded by the delimiter. For example, one can write \# $(\operatorname{tp}(\mathcal{C}(\lambda k$. tp $((\lambda x$. $(\mathcal{T} h k x)+10)(\#(k 3))))+$ $5)$ ), which evaluates to 8 . This does not mean either that any term that behaves like $\mathcal{S}(\lambda q . M)$ must have the form above. For example, $\mathcal{C}(\lambda k \cdot \operatorname{tp}((\lambda x .(\#(k x))+10)(\#(k 3))))$ behaves the same as $\mathcal{S}(\lambda q .((\lambda x . q x+10)(q 3)))$.

\subsection{Dynamic Binding of the Top-Level Continuation}

The discussion and semantics in the previous section explain that the addition of control delimiters amounts to having different bindings to the top-level continuation. To better understand this idea, we propose to modify the original $\lambda_{\mathcal{C} \text { tp }}$ which has a constant corresponding to the top-level continuation by making the top-level continuation a variable. In this section, we explain why this variable cannot be a staticallyscoped variable.

EXAMPLE 5. (Static vs. Dynamic \#) Using the $\lambda_{\mathcal{C} \# \text { tp }}$ reductions, we have (we underline the redex in the first two steps):

$$
\begin{aligned}
& \#\left(\operatorname{tp}\left(\left(\#\left(\operatorname{tp}\left(\lambda_{-} . \mathcal{C}\left(\lambda_{-} . t p\left(\lambda_{-} .3\right)\right)\right)\right)\right)\left(\lambda_{-} \cdot \mathcal{C}\left(\lambda_{-} . t p 4\right)\right)\right)\right) \\
& \left.\rightarrow \#\left(\operatorname{tp}\left(\left(\overline{\left.\lambda_{-} \cdot \mathcal{C}\left(\lambda_{-} . t p\left(\lambda_{-} .3\right)\right)\right)\left(\lambda _ { - } \cdot \mathcal { C } \left(\lambda_{-} . t p\right.\right.} 4\right)\right)\right)\right) \\
& \rightarrow \#\left(\operatorname{tp} \overline{\left.\mathcal{C}\left(\lambda_{-} . \operatorname{tp}\left(\lambda_{-} .3\right)\right)\right)}\right. \\
& \rightarrow \#\left(\operatorname{tp}\left(\lambda_{-} .3\right)\right) \\
& \rightarrow\left(\lambda_{-} .3\right)
\end{aligned}
$$

In the original term, the third occurrence of tp is statically associated with the second occurrence of \#. However, after one reduction step this occurrence of $t p$ is actually associated with the first occurrence of \#. $\mathbf{I}$

Intuitively, a use of a control delimiter introduces a new binding for the top-level continuation for the duration of the evaluation of its subexpression. In other words, the standard semantics of control 


$$
\begin{aligned}
& x, a, v, f, c \in \text { Vars } \\
& k \in \text { StaticKVars } \\
& \text { DynKVars }=\{\widehat{t p}\} \\
& M, N \in \text { Terms }::=x|\lambda x . M| M N|\mathcal{C}(\lambda k . J)| \mathcal{C}(\lambda \widehat{t p} . J) \\
& V \in \text { Values }::=x \mid \lambda x . M \\
& J \in \text { Jumps }::=k M \mid \widehat{t p} M
\end{aligned}
$$

delimiters requires that the binding for the top-level continuation be dynamic (as we will formally prove in the remainder of the paper).

It is possible to use static scope for control delimiters. Not surprisingly, this choice gives a different semantics. Indeed, Thielecke (2002) considers several variations of control operators with different scope rules. He presents a system with static scope and a system with dynamic scope and shows that the system with static scope (adequately extended with infinitely many continuation variables so that capturefree substitution and $\beta$-reduction are correctly defined) corresponds to classical logic and that the system with dynamic scope corresponds to intuitionistic logic. Kameyama (2001) also develops a type system and a semantics for a static variant of control delimiters which he shows has a different semantics than the usual operators.

\subsection{The $\lambda_{\widehat{\mathcal{C}} \widehat{\text { p }}}$-CALCULUS}

As motivated in the previous section the control delimiter can be modelled in an extension of the $\lambda_{\mathcal{C}_{\mathrm{tp}}}$-calculus where the top-level continuation is a dynamically-scoped variable. This extension is given in Figure 9. The grammar now has two distinct uses of $\mathcal{C}$ : one for regular statically-scoped continuation variables and one for the unique dynamically-scoped continuation variable $\widehat{t p}$. We introduce a new abbreviation for an expression which aborts to the closest occurrence of this dynamically-scoped continuation variable:

$$
\widehat{\mathcal{A}} M \triangleq \mathcal{C}\left(\lambda_{-} . \widehat{t p} M\right)
$$

(Abbrev. 3)

The anonymous variable _ ranges only over regular variables and static continuation variables but not over the dynamic variable. Similarly, the notions of free and bound variables only apply to the regular variables and the static continuation variables but not the dynamic variable.

In order to define the semantics of $\lambda_{\mathcal{c t p}}$ we first need an understanding of the semantics of dynamic binding in the absence of control operators (Moreau, 1998). A dynamic abstraction $(\lambda \widehat{x} . M)$ is generally 


\begin{tabular}{|lll|}
\hline$\beta_{v}:$ & $(\lambda x . M) V$ & $\rightarrow M[V / x]$ \\
$\mathcal{C}_{L}:$ & $\mathcal{C}(\lambda k . J) N \rightarrow \mathcal{C}(\lambda k . J[k(\square N) / k])$ \\
$\mathcal{C}_{R}:$ & $V \mathcal{C}(\lambda k . J) \rightarrow \mathcal{C}(\lambda k . J[k(V \square) / k])$ \\
$\mathcal{C}_{\text {idem }}:$ & $\widehat{t p} \mathcal{C}(\lambda k . J) \rightarrow J[\widehat{t p} \square / k]$ \\
$\mathcal{C}_{\text {idem }}:$ & $k^{\prime} \mathcal{C}(\lambda k . J) \rightarrow J\left[k^{\prime} \square / k\right]$ \\
$\mathcal{C}_{\text {elim }}:$ & $\mathcal{C}(\lambda k . k M) \rightarrow M \quad$ where $k \notin F V(M)$ \\
$\mathcal{C}_{\text {elim }}:$ & $\mathcal{C}(\lambda \widehat{t p} . \widehat{t p} V) \rightarrow V \quad$ even if $\widehat{t p}$ occurs in $V$ \\
\hline
\end{tabular}

Figure 10. Reductions of call-by-value $\lambda_{\widehat{\mathcal{C t p}}}$

like a regular function in the sense that when it is called with a value $V$, the formal parameter $\widehat{x}$ is bound to $V$. But:

DS1 The association between $\widehat{x}$ and $V$ established when a function is called lasts exactly as long as the evaluation of the body of the function. In particular, the association is disregarded when the function returns, and this happens even if the function returns something like $\left(\lambda_{-} \ldots \widehat{x} \ldots\right)$ which contains an occurrence of $\widehat{x}$ : no closure is built and the occurrence of $\widehat{x}$ in the return value is allowed to escape.

DS2 The association between $\widehat{x}$ and $V$ introduced by one function may capture occurrences of $\widehat{x}$ that escape from other functions. For example, if we have two dynamic functions $f$ and $g$ with $f$ directly or indirectly calling $g$, then during the evaluation of the body of $f$, occurrences of $\widehat{x}$ that are returned in the result of $g$ will be captured. Turning this example around, the occurrences of $\widehat{x}$ that escape from $g$ are bound by the closest association found up the dynamic chain of calls.

In the presence of control operators, the situation is more complicated because the evaluation of the body of a function may abort or throw to a continuation instead of returning; and capturing a continuation inside the body of a function allows one to re-enter (and hence re-evaluate) the body of the function more than once.

Despite the additional complications, the reduction rules of $\lambda_{\widehat{\mathcal{t p}}}$ in Figure 10 look essentially like the reduction rules of the $\lambda_{\mathcal{C t p}}$-calculus. The noteworthy addition is the rule $\mathcal{C}_{\text {elim }}{ }^{\prime}$ which allows occurrences of $\widehat{t p}$ in $V$ to escape as suggested by DS1 above. Another change, implied by DS2, is that the meta-operation of substitution must allow for the capture of $\widehat{t p}$. The substitution operations $M[V / x]$ and $J[k E / k]$ do not rename $\widehat{t p}$ but are otherwise standard. In particular, we have that:

$$
\mathcal{C}(\lambda \widehat{t p} . J)[M / x] \equiv \mathcal{C}(\lambda \widehat{t p} . J[M / x])
$$

even if $\widehat{t p}$ occurs in $M$. 


$$
\begin{aligned}
& b \in \text { BaseType } \\
& A, B, T \in \text { TypeExp }::=b \mid A \rightarrow B \\
& \Gamma \in \text { Contexts }::=\cdot|\Gamma, x: A| \Gamma, k: A \rightarrow \Perp \\
& \overline{\Gamma, x: A \vdash x: A ; T}^{A} A \\
& \frac{\Gamma, x: A \vdash M: B ; T}{\Gamma \vdash \lambda x \cdot M: A \rightarrow B ; T} \rightarrow_{i} \frac{\Gamma \vdash M: A \rightarrow B ; T \quad \Gamma \vdash M^{\prime}: A ; T}{\Gamma \vdash M M^{\prime}: B ; T} \rightarrow_{e} \\
& \frac{\Gamma, k: A \rightarrow \Perp \vdash J: \Perp ; T}{\Gamma \vdash \mathcal{C}(\lambda k . J): A ; T} R A A \frac{\Gamma \vdash J: \Perp ; T}{\Gamma \vdash \mathcal{C}(\lambda \widehat{t p} . J): T ; T} R A A^{\widehat{t p}} \\
& \frac{\Gamma, k: A \rightarrow \Perp \vdash M: A ; T}{\Gamma, k: A \rightarrow \Perp \vdash k M: \Perp ; T} \rightarrow{ }_{e}^{k} \frac{\Gamma \vdash M: T ; T}{\Gamma \vdash \widehat{t p} M: \Perp ; T} \rightarrow{ }_{e}^{\widehat{t p}}
\end{aligned}
$$

Figure 11. $\Lambda_{\mathcal{C} \widehat{t p}}^{\rightarrow \text { fixed }}$ : a type system of $\lambda_{\widehat{C} \widehat{t p}}$

EXAMPLE 6. (Dynamic capture) The reduction:

$$
\begin{aligned}
& (\lambda x \cdot \lambda y \cdot \mathcal{C}(\lambda \widehat{t p} \cdot \widehat{t p}(x y)))\left(\lambda_{-} \cdot \mathcal{C}\left(\lambda_{-} . \widehat{t p} y\right)\right) \\
& (\lambda y \cdot \mathcal{C}(\lambda \hat{t p} \cdot \widehat{t p}(x y)))\left[\lambda_{-} \mathcal{C}\left(\lambda_{-} . \widehat{t p} y\right) / x\right] \\
& \lambda y^{\prime} \cdot \mathcal{C}\left(\lambda \widehat{t p} \cdot \widehat{t p}\left(\left(\lambda_{-} \cdot \mathcal{C}\left(\lambda_{-} . \widehat{t p} y\right)\right) y^{\prime}\right)\right)
\end{aligned}
$$

captures $\widehat{t p}$ but not $y$.

The exact correspondence between the syntax and reductions of $\lambda_{\mathcal{C} \# \text { tp }}$ and $\lambda_{\widehat{\mathcal{C}} \widehat{\text { tp }}}$ allows to express the following:

PROPOSITION 7. The calculi $\lambda_{\mathcal{C} \# \text { tp }}$ and $\lambda_{\widehat{\mathcal{C t p}}}$ are isomorphic.

\section{The $\lambda_{\widehat{\mathcal{t p}} \widehat{ }}$-Calculus: Static Semantics}

We consider several natural type (and effect) systems for both $\lambda_{\mathcal{C} \# \text { tp }}$ and $\lambda_{\mathcal{C} \widehat{t p}}$ that are inspired by systems in the literature.

\subsection{Type System I: Fixed Answer Type}

The simplest way to produce a type system for $\lambda_{\mathcal{C} \widehat{c p}}$ is to adapt the type system of $\lambda_{\mathcal{C} \text { tp }}$ in minor ways: change the occurrences of tp to be $\widehat{t p}$, and add a new variant of the rule $R A A$ for typing the new use of $\mathcal{C}$. The resulting system is in Figure 11. 
The type system $\Lambda_{\widehat{\mathcal{C} t p}}^{\rightarrow}$ fixed induces the following rule on the $\lambda_{\mathcal{C} \# \text { tp }}$ side:

$$
\frac{\Gamma \vdash M: T ; T}{\Gamma \vdash \#(\operatorname{tp} M): T ; T}
$$

In other words, every occurrence of the control delimiter in the program must be used at the same fixed type $T$. Thus, control operators can only be used in contexts that agree with the top-level type. This situation is similar to the type system one gets when defining control operations on top of a continuation monad with a fixed answer type (Wadler, 1994). Despite its obvious restrictions, this system may be adequate for some applications (Filinski, 1994).

A standard question about type systems is whether they ensure normalisation of typed $\lambda$-terms. It turns out that the type system $\Lambda_{\widehat{\mathcal{C} t p}}^{\rightarrow}$ fixed hides a recursive type in case the top-level type is non-atomic. Strong normalisation is not guaranteed as the following example shows. (See also Proposition 26.)

EXAMPLE 8. (Loss of SN) We present the example using $\lambda_{\mathcal{C A t p}}$. Let $T=($ int $\rightarrow$ int $) \rightarrow$ int be the fixed top-level type, then we can calculate the following types for the given $\lambda_{\mathcal{C} \# \text { tp }}$ expressions:

$$
\begin{array}{ll}
g:: \text { int } \rightarrow T & =\lambda_{\text {_. }} \lambda_{\text {_.0 }} \\
f:: T & =\lambda_{x} .(\#(\operatorname{tp}(g(x)))) x \\
s:: \text { int } \rightarrow \text { int } & =\lambda_{\text {_. }}(\mathcal{A} f) \\
e:: \text { int } & =f s
\end{array}
$$

Despite being well-typed, $e$ goes into an infinite loop:

$$
f s \rightarrow(\#(\operatorname{tp}(g(\mathcal{A} f)))) s \rightarrow(\#(\operatorname{tp} f)) s \rightarrow f s
$$

\subsection{Type System II: Dynamic Binding as an Effect}

The requirement that all occurrences of $\widehat{t p}$ (or equivalently, all occurrences of $\#)$ are typed with the same fixed top-level type $T$ is overly restrictive. Each introduction of $\widehat{t p}$ can be given a different type. This is captured by the following variant of rule $R A A^{\widehat{t p}}$ :

$$
\frac{\Gamma \vdash J: \Perp ; A}{\Gamma \vdash \mathcal{C}(\lambda \widehat{t p} . J): A ; T}
$$




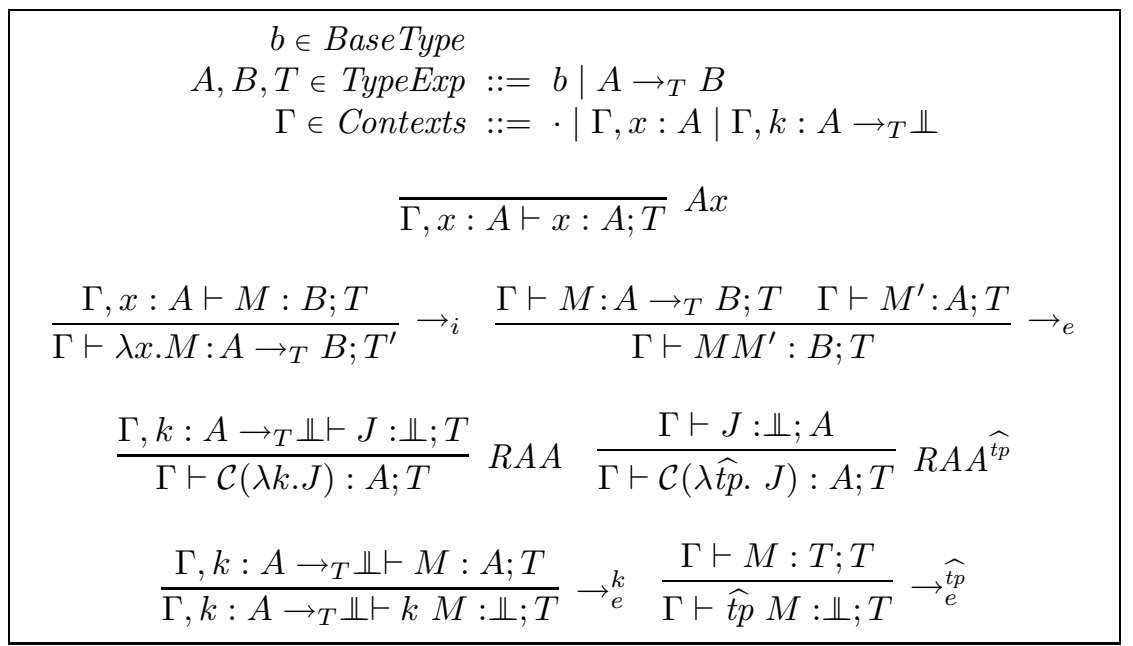

Figure 12. $\Lambda_{\widehat{\mathcal{C t p}}}^{\rightarrow \text { effeq }}$ : a type-and-effect system of $\lambda_{\widehat{C} \widehat{c p}}$

In this rule, if $\widehat{t p}$ is introduced in a context expecting a value of type $A$, then it can be called with arguments of type $A$. In other words, a new top-level type is introduced for the typing of $J$. Thus, the judgement:

$$
\Gamma \vdash M: A ; T
$$

can now be read as "term $M$ returns a value of type $A$ to its immediate context or a value of type $T$ to its enclosing \#." On the $\lambda_{\mathcal{C} \# \text { tp }}$ side, this corresponds to the following rule:

$$
\frac{\Gamma \vdash M: A ; A}{\Gamma \vdash \#(\operatorname{tp} M): A ; T}
$$

This modification is closely related to Murthy's type system (1992).

Unfortunately, this modification is unsound by itself as it changes the type of $\widehat{t p}$ without taking into account that it is dynamically bound. Simply modifying the $R A A^{t p}$ rule of the type system of Figure 11 produces a type system for the static variant of \#: the term considered in Example 5 which evaluates to $\left(\lambda_{-} .3\right)$ is given the type int.

Therefore, in addition to having the modified rule $R A A^{\widehat{t p}}$, we also need to modify the system to take into account that $\widehat{t p}$ is a dynamic variable. A possible modification is to repeat what must be done for other dynamically-bound entities like exceptions (Guzmán and Suárez, 1994), and to add an effect annotation on every arrow type to pass around the type of $\widehat{t p}$. The system with dynamic effect annotations is shown in Figure 12. Using this system the term in Example 5 has type $A \rightarrow_{B}$ int which is consistent with the value $\lambda_{-} .3$. The term 
$\lambda x .(x+\mathcal{A}$ "Hello" $)$ has the type int $\rightarrow$ string int with the string constraint correctly associated with the function call not the function definition.

PROPOSITION 9. Subject reduction: given $\lambda_{\widehat{\mathcal{t p}}}$ terms $M$ and $N$ if $\Gamma \vdash M: A ; T$ in $\Lambda_{\widehat{\mathcal{C} t p}}^{\rightarrow}$ effeq and $M \rightarrow N$ then $\Gamma \vdash N: A ; T$ in $\Lambda_{\widehat{\mathcal{C} t p}}^{\rightarrow \text { effeq }}$.

On the $\lambda_{\mathcal{C} \# \text { tp }}$ side, the corresponding type-and-effect system (which we do not present) addresses the loss of strong normalisation discussed in Example 8. The effect annotations impose the following recursive constraint $T=$ (int $\rightarrow_{T}$ int) $\rightarrow_{T}$ int. In other words, for the terms to typecheck, we must allow recursive type definitions. This is a situation similar to the one described by Lillibridge (1999) where unchecked exceptions can be used to violate strong normalisation. More generally, we can prove that under the type-and-effect system $\Lambda_{\widehat{c} \widehat{t p}}^{\rightarrow \text { effeq }}$ of Figure 12, typed $\lambda_{\widehat{\mathcal{C} t p}}$-terms are strongly normalising. (See Proposition 26.)

INTERMEZZO 10. In the approach discussed above, different occurrences of the symbol \# in a program may have different types. Gunter et al. (1995) take the (quite natural) position that occurrences of \# with different types should have different names. Each name still has a fixed type but that type is not constrained to be the same as the top-level, nor is it constrained to be related to the types of the other names. As in $\Lambda_{\mathcal{C} \widehat{t p}}^{\rightarrow}$ effeq , different occurrences of control delimiters can have different types and the type of the delimiter must be propagated to the control operator. However, it is closer in design to the system with a fixed answer type as all calls to a delimiter of a given name must have the same type. Moreover, since the type system has no effect annotations, Example 8 still typechecks and loops, and well-typed control operations may refer to non-existent control delimiters.

\subsection{Understanding the Dynamic Annotations}

The dynamic annotations we used happen to produce a sound type system, but on closer inspection they are not the "right" annotations. In the following discussion, $\neg \Perp T$ denotes the type of a continuation variable expecting an argument of type $T$, as defined in Section 2.3.

It is standard to embed type systems with effects into regular type systems using a monadic transformation. Since our effect annotations have to do with $\widehat{t p}$ which is a dynamic variable, a first guess would be to use the environment-passing transformation used to explain dynamic scope (Moreau, 1998). At the level of types, the environment-passing 
transformation (written $(\cdot)^{*}$ ) maps $A \rightarrow_{T} B$ to $A^{*} \wedge \neg \Perp T^{*} \rightarrow B^{*}$, which means that every function is passed the (unique) environment binding as an additional argument (we defer to Section 5.1 the question of how to concretely represent the pair of a term and a continuation variable). Judgements $\Gamma \vdash A ; T$ are mapped to $\Gamma^{*}, \neg \Perp T^{*} \vdash A^{*}$ which means that every expression must be typed in the context of its environment. Writing the translation for the pure fragment is easy, but when it comes to $\mathcal{C}$, the translation of the typing rule $R A A$ would produce a continuation whose input type is $A^{*} \wedge \neg \Perp T^{*}$, because continuations also need the environment. But as the rule $R A A$ shows, the input type of the continuation corresponds to the return type of the expression that captures it. In other words, expressions that might capture continuations must return both their value and the environment variable. Thus, our environment-passing embedding becomes a store-passing transformation.

A second interpretation of the annotations would be using exceptions: each delimiter installs an exception handler, and calls to $\widehat{t p}$ throw exceptions to the dynamically-closest handler. Indeed, exceptions can be simulated with ordinary dynamic variables (Moreau, 1998) and with dynamic continuation variables (Gunter et al., 1995). According to the standard monadic interpretation of exceptions (Moggi, 1989), this leads to a transformation mapping $A \rightarrow_{T} B$ to $A^{*} \rightarrow B^{*} \vee T^{*}$, which means that every function may return a value or throw an exception to its delimiter. Judgements $\Gamma \vdash A$; $T$ are mapped to $\Gamma^{*} \vdash A^{*} \vee T^{*}$ and have a similar interpretation. When writing such a translation in a general setting (Thielecke, 2001; Thielecke, 2000; Riecke and Thielecke, 1999), one is faced with a choice: should a use of a control operator capture the current exception handler or not? In our setting, the question is: if a continuation is captured under some \#, and later invoked under another \#, should calls to $\widehat{t p}$ refer to the first \# or the second? It is clear that our semantics requires the second choice, which turns out to be consistent with the SML/NJ control operators capture and escape. In combination with exceptions, these control operators can simulate state (Thielecke, 2001, Fig. 12) which means that our embedding also becomes a store-passing transformation.

The above discussion suggests the following interpretation: functions $A \rightarrow_{T} B$ are mapped to $A^{*} \wedge \neg \Perp T^{*} \rightarrow B^{*} \wedge \neg \Perp T^{*}$ and judgements $\Gamma \vdash A$; $T$ are mapped to $\Gamma^{*}, \neg \Perp T^{*} \vdash A^{*} \wedge \neg \Perp T^{*}$. This interpretation is a standard store-passing one, which is consistent with Filinski's observation that shift and reset can be implemented using continuations and state $(1994,1999)$. The entire analysis is also consistent with the fact that in classical logic, the following formulae are all logically equivalent 


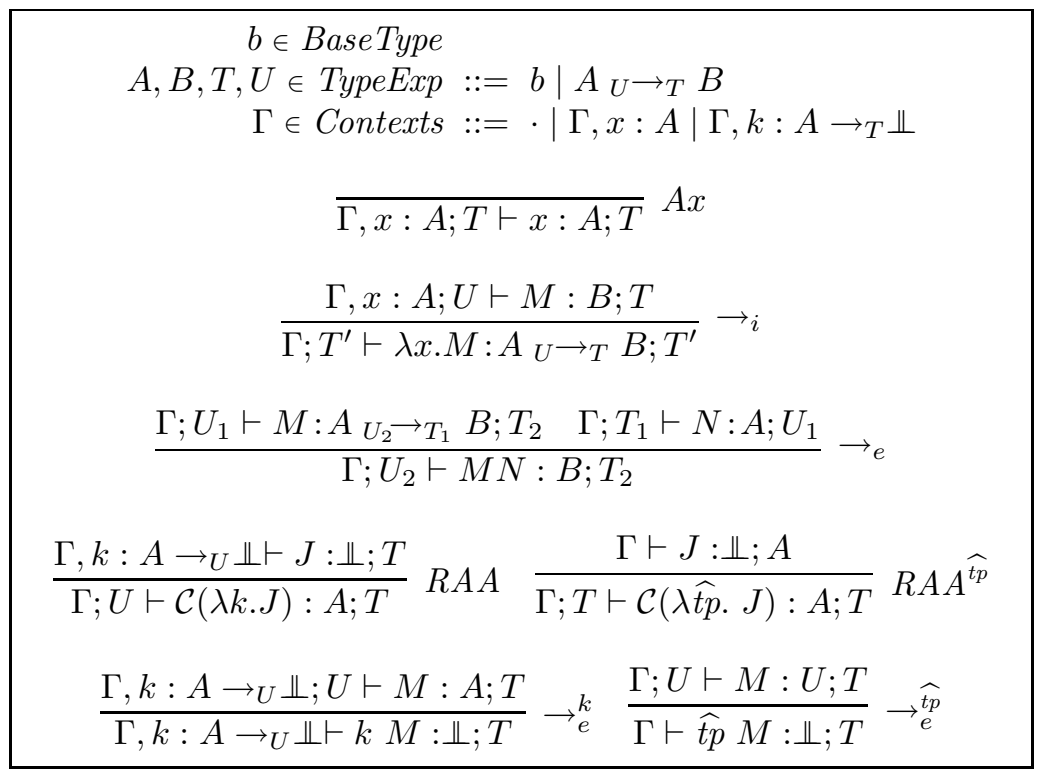

Figure 13. $\Lambda_{\widehat{\mathcal{C} t p}}^{\rightarrow \text { eff }}$ : another type-and-effect system of $\lambda_{\mathcal{C} t p}$

(even though they are not computationally equivalent):

$$
\begin{array}{ll}
A \wedge \neg T \rightarrow B & (\widehat{t p} \text { as an environment }) \\
A \rightarrow B \vee T & (\widehat{t p} \text { as an exception }) \\
A \wedge \neg T \rightarrow B \wedge \neg T & (\widehat{t p} \text { as a state })
\end{array}
$$

\subsection{Type System III: State as Effects}

As the analysis in the previous section shows, $\widehat{t p}$ can be understood as a state parameter and this understanding leads to a different, more expressive, type-and-effect system, which maintains the type of $\widehat{t p}$ before and after each computation. This generalisation gives the type-andeffect system $\Lambda_{\widehat{C} \widehat{t p}}^{\rightarrow}$ eff of Figure 13, which is essentially identical to the one developed by Danvy and Filinski as early as 1989 .

In the cases of jumps and continuations, the judgements and types of the new type system are the same as before. When typing terms, the judgements have the form $\Gamma ; U \vdash M: A ; T$ with $T$ and $U$ describing the top-level continuation before and after the evaluation of the term $M$, respectively. For terms without $\mathcal{C}$, we can show by induction that the two formulae $T$ and $U$ are the same. For terms of the form $\mathcal{C}(\lambda k . J)$, the formula $U$ is the type of the top-level continuation when $k$ is invoked. Implication has two effects $A_{U \rightarrow T} B$ with $T$ describing the top-level continuation before the call and $U$ describing the top-level continuation 
after the call. These changes make the typing of applications sensitive to the order of evaluation of the function and argument: the rule $\rightarrow_{e}$ assumes the function is evaluated before the argument. The new system is sound.

PROPOSITION 11. Subject reduction: given $\lambda_{\widehat{c t p}}$ terms $M$ and $N$, if $\Gamma ; U \vdash M: A ; T$ and $M \rightarrow N$ then $\Gamma ; U \vdash N: A ; T$.

Let $A_{d f}$ be the operation of changing each arrow $B \rightarrow_{T} C$ into the arrow $B \rightarrow_{T} C$ in the formula $A$. Let $\Gamma_{d f}$ be the extension of this operation to $\Gamma$. Let $A_{b}$ be the operation of adding twice the same atomic effect $b$ on the occurrences of $\rightarrow$ in the effect-free formula $A$ (i.e. $B \rightarrow C$ becomes $\left.B{ }_{b} \rightarrow{ }_{b} C\right)$ ). Let $\Gamma_{b}$ be the extension of this operation to an effect-free $\Gamma$. The new type-and-effect system generalises the previous systems.

\section{PROPOSITION 12.}

(i) If $\Gamma \vdash M: A ; T($ resp. $\Gamma \vdash J: \Perp ; T)$ in $\underset{\Lambda_{\mathcal{C f p}}}{\rightarrow \text { effeq }}$ then $\Gamma_{d f} ; T_{d f} \vdash$

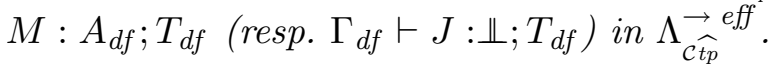

(ii) For atomic $b$, if $\Gamma \vdash M: A ; b$ (resp. $\Gamma \vdash J: \Perp ; b)$ in $\underset{\Lambda_{\mathcal{C t p}}}{\rightarrow \text { fixed }}$ then $\Gamma_{b} ; b \vdash M: A_{b} ; b$ (resp. $\left.\Gamma_{b} \vdash J: \Perp ; b\right)$ in $\underset{\mathcal{C} \widehat{\Lambda_{p}}}{\rightarrow}$ eff

The added expressiveness of the type system is illustrated with the following example.

EXAMPLE 13. The term $\mathcal{C}(\lambda \widehat{t p} \cdot \widehat{t p}(1+\mathcal{C}(\lambda k \cdot \widehat{t p}(2==\mathcal{C}(\lambda \widehat{t p} \cdot k 3)))))$ is rejected by $\Lambda_{\widehat{\mathcal{C} t p}}^{\rightarrow}$ effeq but accepted in $\Lambda_{\widehat{\mathcal{C} t p}}^{\rightarrow}$ eff with the following rule for typing the equality:

$$
\frac{\Gamma ; T_{1} \vdash M: A ; T \quad \Gamma ; U \vdash N: A ; T_{1}}{\Gamma ; U \vdash M==N: \text { bool; } T}
$$

The first occurrence of $k$ is delimited by the outermost occurrence of $\widehat{t p}$ which is of type int, but when $k$ is invoked, the context is delimited by a type bool. Indeed, the term evaluates to a boolean value as shown below:

$$
\begin{aligned}
& \mathcal{C}(\lambda \widehat{t p} \cdot \widehat{t p}(1+\mathcal{C}(\lambda k \cdot \widehat{t p}(2==\mathcal{C}(\lambda \widehat{t p} \cdot k 3))))) \\
\rightarrow & \mathcal{C}(\lambda \widehat{t p} \cdot \widehat{t p}(\mathcal{C}(\lambda k \cdot \widehat{t p}(2==\mathcal{C}(\lambda \widehat{t p} \cdot k(1+3)))))) \\
\rightarrow & \mathcal{C}(\lambda \widehat{t p} \cdot \widehat{t p}(2==\mathcal{C}(\lambda \widehat{t p} \cdot \widehat{t p}(1+3)))) \\
\rightarrow & \mathcal{C}(\lambda \widehat{t p} \cdot \widehat{t p}(2==4)) \rightarrow \text { false }
\end{aligned}
$$


In general, the new type system is expressive enough to give different types to different occurrences of $\widehat{t p}$, as shown in the next example.

EXAMPLE 14. In the term $\mathcal{C}(\lambda \widehat{t p} \cdot \widehat{t p}(\widehat{\mathcal{A}} 5==\widehat{\mathcal{A}}$ "Hello" $))$ the visible occurrence of $\widehat{t p}$ and the two implicit occurrences in $\widehat{\mathcal{A}}$ have the three different types: bool, int and string respectively, as indicated in the derivation below.

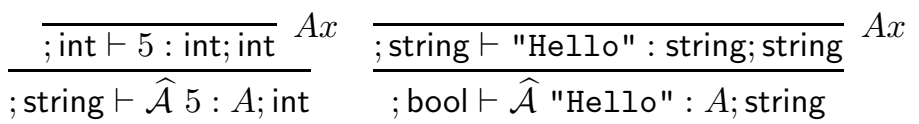

$$
\begin{aligned}
& \frac{; \text { bool } \vdash \widehat{\mathcal{A}} 5==\widehat{\mathcal{A}} \text { "Hello" : bool; int }}{\vdash \widehat{t p}(\widehat{\mathcal{A}} 5==\widehat{\mathcal{A}} \text { "Hello" }): \mathbb{\Perp} ; \text { int }} \rightarrow_{e}^{p p} \\
& \frac{\vdash t p(\widehat{\mathcal{A}} 5==\widehat{\mathcal{A}} \text { "Hello" }): \Perp ; \text { int }}{; T \vdash \mathcal{C}(\lambda \widehat{t p} . \widehat{t p}(\widehat{\mathcal{A}} 5==\widehat{\mathcal{A}} \text { "Hello" })): \operatorname{int} ; T} R A A^{\widehat{t p}}
\end{aligned}
$$

\section{The $\lambda_{\mathcal{C}}^{-}$-Calculus}

We show that the dual connective of implication, namely subtraction (written $A-B$ ) arises as the natural type for carrying around the type of the top-level continuation, and formally introduce the $\lambda_{\mathcal{C}}^{-}$-calculus.

\subsection{Subtraction}

Our analysis in Section 4.3 together with our understanding of the

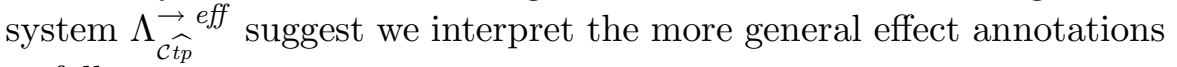
as follows:

$$
\begin{aligned}
\left(A_{U \rightarrow T} B\right)^{*} & =A^{*} \wedge \neg \Perp T^{*} \rightarrow B^{*} \wedge \neg \Perp U^{*} \\
(\Gamma ; U \vdash A ; T)^{*} & =\Gamma^{*}, \neg \Perp T^{*} \vdash A^{*} \wedge \neg \Perp U^{*}
\end{aligned}
$$

But the question arises: how to represent $\neg \Perp T^{*}$ as a type? A natural attempt is to embed $\neg \Perp T^{*}$ into the type of functions $T^{*} \rightarrow \perp$ as shown in Section 2.3, but it is also possible to investigate a more abstract solution based on the subtraction connective.

The subtractive type has been previously studied by Rauszer (1974) and Crolard (2001), and has been integrated by Curien and Herbelin (2000) in their study of the duality between the producers of values (which are regular terms) and the consumers of values (which are contexts or continuations). Subtraction has been also considered in Filinski (1989) where it is named a co-exponential. In particular, Filinski 


$\begin{array}{|ll|}b^{*} & =b \\ \left(A_{U \rightarrow T} B\right)^{*} & =\left(A^{*}-T^{*}\right) \rightarrow\left(B^{*}-U^{*}\right) \\ (\cdot)^{*} & =\cdot \\ (\Gamma, x: A)^{*} & =\Gamma^{*}, x: A^{*} \\ (\Gamma, k: A \rightarrow U \Perp)^{*} & =\Gamma^{*}, k:\left(A^{*}-U^{*}\right) \rightarrow \Perp\end{array}$

Figure 14. Interpreting the effect annotations

$$
\begin{aligned}
& \frac{\Gamma \vdash \Delta, C ; A \quad \Gamma, B \vdash \Delta, C ; C}{\Gamma \vdash \Delta, C ; A-B}-i \\
& \frac{\Gamma \vdash \Delta ; A-B \quad \Gamma, A \vdash \Delta ; B}{\Gamma \vdash \Delta ; C}-e
\end{aligned}
$$

Figure 15. Subtractive logic in Parigot's style (classical natural deduction)

presents a duality between functions of type $A \rightarrow B$ and continuations of type $A-B$ (there written $[B \leftarrow A]$ ) that are interpreted as continuation transformers. In our work, the subtraction arises as the natural type for pairing a term and an evaluation context and we use it as a direct representation of this information rather than to rely on an embedding of evaluation contexts (of type $\neg \Perp T$ ) into functions (of type $\neg T)$.

The actual interpretation of effects we use is in Figure 14. (The correctness of the interpretation is discussed in Section 6 after we explain the target of the translation in detail.) The interpretation shows that a continuation $k$ has type $(A-T) \rightarrow \Perp$. Understanding $A-T$ as the type $A \wedge \neg T$ means that, as Danvy and Filinski explain, every delimited continuation must be given a value and another continuation (called the metacontinuation in their original article (Danvy and Filinski, 1990) and referring to the top-level continuation as we explain). The type $A-T$ is also equivalent to $\neg(\neg T \rightarrow \neg A)$ and with that view, a delimited continuation is more like a continuation transformer, which is an idea closely related to Queinnec and Moreau's formalisation of delimited continuations as the difference between two continuations (1994).

The embedding of a function type is of the form $(A-T) \rightarrow(B-U)$ which captures the idea that the top-level type $T$ needed to execute the function's body should be available when the function is called, and that the new top-level type $U$ is returned as part of the result.

INTERMEZZO 15.

Figure 15 presents the subtraction rules introduced by Crolard (2004) in the style of Parigot's classical natural deduction. In that setting one works with two sets of assumptions: $\Gamma$ and $\Delta$, where $\Delta$ maintains the 


$$
\begin{gathered}
A, B::=X|A \rightarrow B| A-B \\
\Gamma::=\cdot|\Gamma, A| \Gamma, A \rightarrow \Perp \\
\frac{\Gamma, A \vdash A}{\Gamma x} \\
\frac{\Gamma, A \vdash B}{\Gamma \vdash A \rightarrow B} \rightarrow_{i} \frac{\Gamma \vdash A \rightarrow B \quad \Gamma \vdash A}{\Gamma \vdash B} \rightarrow_{e} \\
\frac{\Gamma, A \rightarrow \Perp \vdash \Perp}{\Gamma \vdash A} R A A \quad \frac{\Gamma, A \rightarrow \Perp \vdash A}{\Gamma, A \rightarrow \Perp \vdash \Perp} \rightarrow_{e}^{k} \\
\frac{\Gamma \vdash A \quad \Gamma, B \vdash \Perp}{\Gamma \vdash A-B}-i \frac{\Gamma \vdash A-B \quad \Gamma, A, B \rightarrow \Perp \vdash \Perp}{\Gamma \vdash \Perp}-e
\end{gathered}
$$

Figure 16. Classical subtractive logic

continuation variables. In a setting with continuation variables written on the left-hand side of the sequent, the natural representation of $\Delta=\left\{A_{1}, \cdots, A_{n}\right\}$ is as $\neg \Perp \Delta$ defined as $\left\{\neg \Perp A_{1}, \cdots, \neg \Perp A_{n}\right\}$. With this notation, the subtraction rules rewrite to:

$$
\begin{aligned}
& \frac{\Gamma, \neg \Perp \Delta, \neg \Perp C \vdash A \quad \Gamma, B, \neg \Perp \Delta, \neg \Perp C \vdash C}{\Gamma, \neg \Perp \Delta, \neg \Perp C \vdash A-B}-{ }_{i} \\
& \frac{\Gamma, \neg \Perp \Delta \vdash A-B \quad \Gamma, A, \neg \Perp \Delta \vdash B}{\Gamma, \neg \Perp \Delta \vdash C}-{ }_{e}
\end{aligned}
$$

Figure 16 presents our variant of the classical natural deduction with subtraction. A formula is built from a set of atomic formulae (X), which we leave unspecified, and two logical connectives. We make use of two kinds of judgements: $\Gamma \vdash A$ and $\Gamma \vdash \Perp$. The rules for subtraction are slightly different. We can recover Crolard's rules as follows:

$$
\begin{gathered}
\Gamma, \neg \Perp \Delta, \neg \Perp C \vdash A \quad \frac{\Gamma, B, \neg \Perp \Delta, \neg \Perp C \vdash C}{\Gamma, B, \neg \Perp \Delta, \neg \Perp C \vdash \Perp} \rightarrow_{e}^{k} \\
\frac{{ }_{i}}{\Gamma, \neg \Perp \Delta, \neg \Perp C \vdash A-B} \\
\frac{\Gamma, \neg \Perp \Delta, \neg \Perp C \vdash A-B \quad \frac{\Gamma, \neg \Perp \Delta, \neg \Perp B, A, \neg \Perp C \vdash B}{\Gamma, \neg \Perp \Delta, \neg \Perp B, A, \neg \Perp C \vdash \Perp}}{\frac{\Gamma, \neg \Perp \Delta, \neg \Perp C \vdash \Perp}{\Gamma, \neg \Perp} R A A}-{ }_{e}^{k} \\
e_{e}^{k}
\end{gathered}
$$




$$
\begin{aligned}
& x, a, v, f \in \text { Vars } \\
& k, t p \in \text { KVars } \\
& M, N \in \text { Terms }::= x|\lambda x . M| M N|\mathcal{C}(\lambda k . J)| \\
&\left(M, \lambda x . J_{c}[E[x]]\right) \\
& V \in \text { Values }::=x|\lambda x . M|\left(V, \lambda x . J_{c}[E[x]]\right) \\
& J \in \text { Jumps }::=k M \mid \text { let }(x, k)=M \text { in } J \\
& J_{c} \in \text { ElemJumpCtx }::=k \square \mid \text { let }(x, k)=\square \text { in } J \\
& F \in \text { ElemCtxt }::=\square M|V \square|\left(\square, \lambda x . J_{c}[E[x]]\right) \\
& E \in \text { EvCtxt }::=\square \mid E[F]
\end{aligned}
$$

Figure 17. Syntax of $\lambda_{\mathcal{C}}^{-}$

\subsection{Syntax, Semantics, and Type System}

We formalise the $\lambda_{\mathcal{C}}^{-}$-calculus by extending the original $\lambda_{\mathcal{C} \text { tp }}$ with subtraction and removing the special constant tp since we are maintaining the top-level continuation using the subtractive type. The resulting calculus is a call-by-value variant of Crolard's extension of the $\lambda \mu$-calculus with subtraction (2004).

The syntax is given in Figure 17. Subtractions are introduced by terms of the form $(M, \lambda x . k E[x])$ or $\left(M, \lambda x\right.$. let $\left(x^{\prime}, k\right)=E[x]$ in $\left.J\right)$. We represent them both using the syntactic category $J_{c}$ and write $\left(M, \lambda x . J_{c}[E[x]]\right)$. We call the second component of the pair a jump context. The use of a jump context in place of a continuation variable is motivated and explained below. The notation $\lambda x . J_{c}[E[x]]$ is basically a writing for the pair of the elementary jump context $J_{c}$ and of the evaluation context $E$. The role of the abstraction over $x$ is purely decorative: it is a way to recall that the hole of $J_{c}[E]$ is bound in the pair $\left(M, \lambda x . J_{c}[E[x]]\right)$. In particular, in the evaluation context $\left(E^{\prime}, \lambda x . J_{c}[E[x]]\right)$, the hole is in $E^{\prime}$, not in $E$. As an abuse of notation, we write $k$ as an abbreviation for the jump context $\lambda x$. $k x$. Subtractions are eliminated by a jump of the form let $(x, k)=M$ in $J$. The notation $E[F]$ is pure syntax: it denotes the pair of $E$ and $F$. Contrastingly, each of the notations $F[M]$ and $E[M]$ (resp. $J_{c}[M]$ and $J_{c}[E[M]]$ ) that are used later on denote the term (resp. jump) obtained by interpreting the context as a partial term (resp. jump) and by filling the hole of this partial term with $M$.

The reduction semantics is given in Figure 18. The $\mathcal{C}_{\text {lift }}$ rule lifts a $\mathcal{C}$-expression out of any of the three possible elementary contexts $F$ : these include applicative contexts as before as well as contexts of the form $\left(\square, \lambda x . J_{c}[E[x]]\right)$.

Consider the $\lambda_{\widehat{\mathcal{t p}}}$ term $\mathcal{C}(\lambda k . \widehat{t p}(\mathcal{T h} k 2))$. After the invocation of continuation $k$, control goes back to the current top-level continuation 


$$
\begin{aligned}
& \beta_{v}: \quad(\lambda x \cdot M) V \quad \rightarrow M[V / x] \\
& \mathcal{C}_{\text {lift }:} \quad F[\mathcal{C}(\lambda k . J)] \quad \rightarrow \mathcal{C}(\lambda k . J[k F / k]) \\
& \mathcal{C}_{\text {elim }}: \quad \mathcal{C}(\lambda k . k M) \quad \rightarrow M \text { where } k \notin F V(M) \\
& \mathcal{C}_{\text {idem }}: \quad k^{\prime} \mathcal{C}(\lambda k . J) \quad \rightarrow J\left[k^{\prime} \square / k\right] \\
& \text { Sub } b_{v}^{\text {lift }}: \quad \text { let }(x, k)=\mathcal{C}\left(\lambda k^{\prime} . J^{\prime}\right) \quad \rightarrow J^{\prime}\left[\operatorname{let}(x, k)=\square \text { in } J / k^{\prime}\right] \\
& S u b_{v}^{\text {base }}: \operatorname{let}(x, k)=\left(V, \lambda x . J_{c}[x]\right) \quad \rightarrow J\left[J_{c} / k ; V / x\right] \\
& \text { in } J \\
& S u b_{v}^{\text {step }}: \quad \text { let }(x, k)=\left(V, \lambda x . J_{c}[E[F[x]]]\right) \rightarrow \text { let }(x, k)=\left(V, \lambda x . J_{c}[E[x]]\right) \\
& \text { in } J \quad \text { in } J[k F / k]
\end{aligned}
$$

Figure 18. Reductions of call-by-value $\lambda_{\mathcal{C}}^{-}$

bound to $\widehat{t p}$. If this top-level continuation has value $t p$, then we can express the example using subtraction by writing $\mathcal{C}(\lambda k . k(2, t p))$. In $\lambda_{\mathcal{C}}^{-}$, the dynamically-scoped variable $\widehat{t p}$ disappears. Instead, the invocation of $k$ is given the current top-level context which must be invoked next. In this particular case, the jump context is essentially just a continuation variable but things can be more complicated.

As another example, consider the term $\mathcal{C}(\lambda k \cdot \widehat{t p}(\mathcal{C}(\lambda \widehat{t p} . k 2)==3))$. After the invocation of continuation $k$, control returns to the context $\square==3$ and then to the top-level continuation bound to $\widehat{t p}$. If this top-level continuation has value $t p$, then we can express the example using subtraction by writing $\mathcal{C}(\lambda k . k(2, \lambda x . t p(x==3)))$. In this case, the jump context is not simply a continuation variable, indicating that the continuation composes with another context before returning to the top-level.

To see this behaviour simulated by the reduction rules, consider a jump expression which eliminates the subtraction and invokes the continuation:

$$
\text { let }(x, k)=(2, \lambda x \cdot \operatorname{tp}(x==3)) \text { in } k x
$$

Using $S u b_{v}^{\text {step }}$, the context $\square==3$ is lifted:

$$
\begin{array}{ll}
\text { let }(x, k)=(2, \lambda x \cdot t p(x==3)) \rightarrow & \text { let }(x, k)=(2, t p) \\
\text { in } k x & \text { in } k(x==3)
\end{array}
$$

and then using $S u b_{v}^{\text {base }}$ control is transferred to the top-level:

$$
\begin{aligned}
& \text { let }(x, k)=(2, t p) \rightarrow t p(2==3) \\
& \text { in } k(x==3)
\end{aligned}
$$

In general the jump context may include an arbitrary nesting of elementary contexts. For example, we can have:

$$
\begin{aligned}
& \text { let }(x, k)=\left(z, \lambda x \cdot \operatorname{tp}\left(\left(\left(x N_{1}\right) N_{2}\right) N_{3}\right)\right) \\
& \text { in } k x
\end{aligned}
$$


The question arises whether it is more convenient or natural to lift the context $\left(\left(\square N_{1}\right) N_{2}\right) N_{3}$ in one step or in many steps in the style of the rule $\mathcal{C}_{\text {lift }}$. We adopt a one by one lifting of $\square N_{1}$, $\square N_{2}$ and $\square N_{3}$ as shown below:

$$
\begin{aligned}
& \text { let }(x, k)=\left(z, \lambda x \cdot \operatorname{tp}\left(\left(\left(x N_{1}\right) N_{2}\right) N_{3}\right)\right) \text { in } k x \\
& \rightarrow \text { let }(x, k)=\left(z, \lambda x \cdot t p\left(\left(x N_{2}\right) N_{3}\right)\right) \text { in } k\left(x N_{1}\right) \\
& \rightarrow \text { let }(x, k)=\left(z, \lambda x \cdot \operatorname{tp}\left(x N_{3}\right)\right) \text { in } k\left(\left(x N_{1}\right) N_{2}\right) \\
& \rightarrow \text { let }(x, k)=(z, t p) \text { in } k\left(\left(\left(x N_{1}\right) N_{2}\right) N_{3}\right) \\
& \rightarrow \operatorname{tp}\left(\left(\left(z N_{1}\right) N_{2}\right) N_{3}\right)
\end{aligned}
$$

The above discussion motivates jump contexts of the form $\lambda x . k E[x]$. The form $\lambda x$. let $\left(x^{\prime}, k\right)=E[x]$ in $J$ is motivated by the following $S u b_{v}^{\text {lift }}$-reduction:

$$
\begin{aligned}
& \text { let }\left(x^{\prime}, k\right)=\mathcal{C}\left(\lambda q . k^{\prime}(2, \lambda x . q x)\right) \rightarrow k^{\prime}\left(2, \lambda x \text {. let }\left(x^{\prime}, k\right)=x\right. \\
& \text { in } \left.k x^{\prime} \quad \text { in } k x^{\prime}\right)
\end{aligned}
$$

INTERMEZZO 16.

In Section 2.3, we showed how to embed $\neg \Perp T$ into $\neg T$. For the other direction, we need to explicitly consider an elimination rule for $\perp$ :

$$
\frac{\Gamma \vdash M: \perp}{\Gamma \vdash \operatorname{tp}_{\perp} M: \Perp} \perp_{e}
$$

The continuation $\mathrm{tp}_{\perp}$ is the constant tp considered in Section 2 restricted to the $\perp$ type: it is the dual of the unique inhabitant of the unit type $T$. Using $\mathrm{tp}_{\perp}$, any term $M$ of type $T \rightarrow \perp$ can be turned into the jump context $\lambda x \cdot \operatorname{tp}_{\perp}((\lambda y \cdot M y) x)$.

The following abbreviations capture some common patterns:

$$
\begin{aligned}
\lambda(x, k) \cdot M & \triangleq \lambda v \cdot \mathcal{C}(\lambda q \cdot \text { let }(x, k)=v \text { in } q M) \\
\text { join } M & \triangleq \operatorname{let}(x, k)=M \text { in } k x \\
\text { bind }_{q, k} M \text { in } N & \triangleq \operatorname{let}(f, k)=M \text { in } q(f N)
\end{aligned}
$$

In the first abbreviation $v$ is a fresh variable. The operator join abbreviates the common pattern where the elimination form of the subtractive value immediately throws the value to the jump context. The operator bind is similar to the monadic operator of the same name. Both $M$ and $N$ are expected to be terms that evaluate to subtractive values with $N$ containing free occurrences of $k$ and $f$ is a fresh variable: the effects of $M$ are performed to produce a subtractive value which is bound to $(f, k)$ and then $f$ is applied to $N$.

The typing rules for the complete language are in Figure 19. The system named $\Lambda_{\mathcal{C}}-$ is completely standard with no effect annotations 


$$
\begin{gathered}
b \in \text { BaseType } \\
A, B, C, T, U \in \text { TypeExp }::=b|A \rightarrow B| A-B \\
\Gamma \in \text { Contexts }::=\cdot|\Gamma, x: A| \Gamma, k: A \rightarrow \Perp \\
\frac{\Gamma, x: A \vdash x: A}{\Gamma} A x \\
\frac{\Gamma, x: A \vdash M: B}{\Gamma \vdash \lambda x . M: A \rightarrow B} \rightarrow_{i} \quad \frac{\Gamma \vdash M: A \rightarrow B \quad \Gamma \vdash M^{\prime}: A}{\Gamma \vdash M M^{\prime}: B} \rightarrow_{e} \\
\frac{\Gamma, k: A \rightarrow \Perp \vdash J: \Perp}{\Gamma \vdash \mathcal{C}(\lambda k . J): A} R A A \quad \frac{\Gamma, k: A \rightarrow \Perp \vdash M: A}{\Gamma, k: A \rightarrow \Perp \vdash k M: \Perp} \rightarrow_{e}^{k} \\
\frac{\Gamma \vdash M: A \quad \Gamma, x: B \vdash J_{c}[E[x]]: \Perp}{\Gamma \vdash\left(M, \lambda x . J_{c}[E[x]]\right): A-B}-{ }_{i} \\
\frac{\Gamma \vdash M: A-B \quad \Gamma, x: A, k: B \rightarrow \Perp \vdash J: \Perp}{\Gamma \vdash \operatorname{let}(x, k)=M \text { in } J: \Perp}-{ }_{e}
\end{gathered}
$$

Figure 19. $\Lambda_{\mathcal{C}}^{-}$: type system of $\lambda_{\mathcal{C}}^{-}$

and not even a global parameter $T$. Note that the right premise of the rule $-{ }_{i}$ must be read as "there is a derivation of $\Gamma, x: B \vdash J: \Perp$ for $J$ obtained by filling the jump context $J_{c}$ with $E[x]$ where $x$ is chosen such that it does not occur in $J_{c}$ nor in $E$ ".

\section{PROPOSITION 17.}

1. Subject reduction: Given $\lambda_{\mathcal{C}}^{-}$terms $M$ and $N$ if $\Gamma \vdash M: A$ and $M \rightarrow N$ then $\Gamma \vdash N: A$.

2. Typed $\lambda_{\mathcal{C}}^{-}$terms are strongly normalising.

Proof. Subject reduction is routine verification. The proof of strong normalisation is expanded in detail in Appendix A.

\section{Embeddings in $\lambda_{\mathcal{C}}^{-}$}

We introduce the full embedding of $\lambda_{\widehat{c} \widehat{t p}}$ into $\lambda_{\mathcal{C}}^{-}$and show its correctness. Our starting point is the effect logic of Figure 20 which gives the type judgements of $\lambda_{\widehat{C} \widehat{t p}}$ with no terms. The aim is to embed such 


$$
\begin{gathered}
A, B, T, U::=X \mid A_{U \rightarrow T} B \\
\Gamma::=\cdot|\Gamma, A| \Gamma, A \rightarrow_{T} \Perp \\
\frac{\Gamma, A ; T \vdash A ; T}{\Gamma x} \\
\frac{\Gamma, A ; U \vdash B ; T}{\Gamma ; T^{\prime} \vdash: A_{U} \rightarrow_{T} B ; T^{\prime}} \rightarrow_{i} \frac{\Gamma ; U_{1} \vdash A_{U_{2} \rightarrow_{1}} B ; T_{2} \quad \Gamma ; T_{1} \vdash A ; U_{1}}{\Gamma ; U_{2} \vdash B ; T_{2}} \rightarrow_{e} \\
\frac{\Gamma, A \rightarrow_{U} \Perp \vdash \Perp ; T}{\Gamma ; U \vdash A ; T} R A A \quad \frac{\Gamma \vdash \Perp ; A}{\Gamma ; T \vdash A ; T} R A A^{\widehat{t p}} \\
\frac{\Gamma, A \rightarrow_{U} \Perp ; U \vdash A ; T}{\Gamma, A \rightarrow_{U} \Perp \vdash \Perp ; T} \rightarrow_{e}^{k} \frac{\Gamma ; U \vdash U ; T}{\Gamma \vdash \Perp} \rightarrow_{e}^{\widehat{t p}}
\end{gathered}
$$

Figure 20. Natural deduction with effects

judgements into the judgements of the subtractive logic of Figure 16, which is a term-free version of the type system of $\lambda_{\mathcal{C}}^{-}$:

$$
\begin{aligned}
& (\Gamma ; B \vdash A ; C)^{*}=\Gamma^{*}, \neg \Perp C^{*} \vdash A^{*}-B^{*} \\
& (\Gamma \vdash \Perp ; C)^{*}=\Gamma^{*}, \neg \Perp C^{*} \vdash \Perp
\end{aligned}
$$

The embedding extends the embedding of Figure 14 which maps $\lambda_{\widehat{\mathcal{C}} \widehat{p}}$ types and contexts into $\lambda_{\mathcal{C}}^{-}$.

When extended with a term assignment, the embeddings become:

$$
\begin{aligned}
& (\Gamma ; B \vdash M: A ; C)^{t p}=\Gamma^{*}, t p: \neg \Perp C^{*} \vdash M^{t p}: A^{*}-B^{*} \\
& (\Gamma \vdash J: \Perp ; C)^{t p}=\Gamma^{*}, t p: \neg \Perp C^{*} \vdash J^{t p}: \Perp
\end{aligned}
$$

where the value $t p$ of the dynamic top-level continuation is required as a parameter.

Figure 21 summarises the embedding of terms and jumps. The translation in the figure includes a special translation $(\cdot)^{+}$on values with the following property. The translation of a value has no free occurrences of the continuation variable used in the embedding. More precisely, every function takes a subtractive value as an argument which specifies the original argument and the top-level continuation resulting from the evaluation of the function and the argument. This allows us to infer for example that in the special case where we jump to the top-level with a value, we have $(\widehat{t p} V)^{t p} \rightarrow t p V^{+}$. We further discuss some cases of the embedding below.

In the translation of an application of the form $M N$, for $M$ not a value, the references to the top-level in $N^{t p}$ are bound by the bind $\mathbf{b}_{q, t p}$, while the occurrences of the top-level in $M^{t p}$ are free. These relationships mimic the fact that the actual binding for a call to $\widehat{t p}$ in $N$ is 


$$
\begin{aligned}
x^{+} & =x \\
(\lambda x . M)^{+} & =\lambda(x, t p) \cdot M^{t p} \\
V^{t p} & =\left(V^{+}, t p\right) \\
(V N)^{t p} & =V^{+} N^{t p} \\
(M N)^{t p} & =\mathcal{C}(\lambda q \cdot \text { bind } \\
q, t p & \left.M^{t p} \text { in } N^{t p}\right) \quad(M \text { not a value }) \\
(\mathcal{C}(\lambda k . J))^{t p} & =\mathcal{C}\left(\lambda k . J^{t p}\right) \\
(\mathcal{C}(\lambda \widehat{t p} . J))^{t p} & =\left(\mathcal{C}\left(\lambda t p . J^{t p}\right), t p\right) \\
(k M)^{t p} & =k M^{t p} \\
(\widehat{t p} M)^{t p} & =\text { join } M^{t p}
\end{aligned}
$$

Figure 21. Embedding of $\lambda_{\widehat{\mathcal{C t p}}}$ terms and jumps into $\lambda_{\mathcal{C}}^{-}$

the one active when $M$ returns its result, whereas the actual binding for a call to $\widehat{t p}$ in $M$ is the one at the time of evaluating $M N$. The embedding of $\mathcal{C}(\lambda \widehat{t p} . J)$ returns a subtractive term with the current top-level continuation tp as the jump context and a computation which introduces a fresh tp thus simulating the rebinding of the top-level continuation in $J$. Note that $t p$ does not expect a subtractive value as its argument. Thus, the argument passed to a top-level jump is evaluated to produce a value and a jump context, which are consumed before returning to $t p$.

\subsection{Soundness of THE EMBEDding OF $\lambda_{\widehat{\mathcal{C}} \widehat{\text { p }}}$ INTO $\lambda_{\mathcal{C}}^{-}$}

For $t p$ a fresh continuation variable, the embedding is sound in the sense that it maps judgements of $\underset{\Lambda_{\widehat{C} t p}}{\rightarrow}$ eff (Figure 13) to judgements of $\Lambda_{\mathcal{C}}-$ (Figure 19).

\section{PROPOSITION 18.}

(i) If $\Gamma ; U \vdash M: A ; T$ in $\underset{\widehat{\mathcal{C} t p}}{\rightarrow}$ eff then $(\Gamma ; U \vdash M: A ; T)^{t p}$ in $\Lambda_{\mathcal{C}}^{-}$,

(ii) If $\Gamma \vdash J: \Perp ; T$ in $\underset{\mathcal{C} \widehat{t p}}{\rightarrow}$ eff then $(\Gamma \vdash J: \Perp ; T)^{t p}$ in $\Lambda_{\mathcal{C}}^{\rightarrow-}$.

Based on Proposition 12, the soundness argument also extends to the type system $\Lambda_{\mathcal{C} \widehat{t p}}^{\rightarrow \text { effeq }}$ (Figure 12) and $\Lambda_{\widehat{\mathcal{C t p}}}^{\rightarrow \text { fixed }}$ (Figure 11) for $\lambda_{\widehat{\mathcal{C} t p}}$.

\section{PROPOSITION 19.}

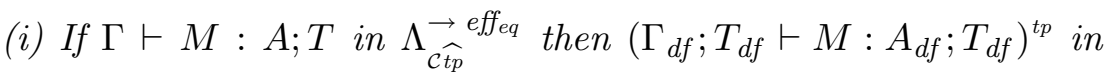
$\Lambda_{\mathcal{c}}-$. 
(ii) If $\Gamma \vdash M: A ; b$ in $\underset{\mathcal{C} \widehat{\mathcal{C t p}}}{\overrightarrow{\text { fixed }}}$ then $\left(\Gamma_{T} ; b \vdash M: A_{b} ; b\right)^{t p}$ in $\Lambda_{\mathcal{C}}^{\overrightarrow{-}}$ for an atomic type $b$.

The embedding is also sound with respect to the semantics. More precisely, each reduction step in $\lambda_{\widehat{c} \widehat{t p}}$ maps to a reduction sequence of at least one step (written $\rightarrow \geq 1$ ) in $\lambda_{\mathcal{C}}^{-}$up to some renaming steps which are carried out by the $\mathcal{C}_{\text {idem }}$ rule. The reason for these renaming steps is due to the administrative redexes introduced by the translation as shown in the example below. Consider the following reduction in $\lambda_{\widehat{\mathcal{C t p}}}$ :

$$
\mathcal{C}(\lambda k . k N) M \rightarrow \mathcal{C}(\lambda k . k(N M))
$$

For simplicity we assume $k$ does not occur free in $N$. The embedding of the left-hand side reduces to

$$
\mathcal{C}\left(\lambda q . \text { let }\left(f, t p^{\prime}\right)=N^{t p} \text { in } q\left(f M^{t p^{\prime}}\right)\right)
$$

whereas the embedding of the right-hand side is:

$$
\mathcal{C}\left(\lambda k . k \mathcal{C}\left(\lambda q . \text { let }\left(f, t p^{\prime}\right)=N^{t p} \text { in } q\left(f M^{t p^{\prime}}\right)\right)\right)
$$

Before stating the soundness we need a lemma indicating how the embedding behaves with respect to substitution. To that end, we define the following abbreviations:

$$
\begin{aligned}
& (k(\square M))^{+}=\text {bind }_{k, t p} \square \text { in } M^{t p} \\
& (k(V \square))^{+}=k\left(V^{+} \square\right) \\
& (\widehat{t p} \square)^{+}=\text {join } \square
\end{aligned}
$$

LEMMA 20. The following properties hold:

(i) $M^{t p}\left[V^{+} / x\right]=(M[V / x])^{t p}$,

(ii) $(J[k(V \square) / k])^{t p}=J^{t p}\left[(k(V \square))^{+} / k\right]$,

(iii) $(J[q(\square M) / k])^{t p} \rightarrow \mathcal{C}_{\text {idem }} N \leftarrow J^{t p}\left[(q(\square M))^{+} / k\right]$ for some $N$,

(iv) $(J[\widehat{t p} \square / k])^{t p}=J^{t p}\left[(\widehat{t p} \square)^{+} / k\right]$.

Proof. The non-obvious part is item (iii). The translation of each subterm $k(N M)$ in $J[k(\square M) / k]$ depends on whether $N$ is a value or not. If $N$ is some value $V$, then $k(V M)$ is translated to $k\left(V^{+} M^{t p}\right)$ which is a contractum of $\mathbf{b i n d}_{k, t p} V^{t p}$ in $M^{t p}$. If $N$ is not a value, the translation is $k \mathcal{C}\left(\lambda q\right.$. bind $_{q, t p}, N^{t p}$ in $\left.M^{t p}\right)$ which reduces by $\mathcal{C}_{\text {idem }}$ to bind $_{k, t p} N^{t p}$ in $M^{t p}$. 
PROPOSITION 21. If $M \rightarrow N$ in $\lambda_{\widehat{c} \widehat{t p}}$ then there exists $N^{\prime}$ such that $M^{t p} \rightarrow{ }^{\geq 1} N^{\prime}$ in $\lambda_{\mathcal{C}}^{-}$and $N^{t p} \rightarrow \mathcal{C}_{\text {idem }} N^{\prime}$.

Proof. We show one case of the proof:

Case $\mathcal{C}_{L}$ :

$$
\begin{array}{ll} 
& \left(\mathcal{C}\left(\lambda k^{\prime} . J\right) N\right)^{t p}=\mathcal{C}\left(\lambda q . \text { bind }_{q, t p} \mathcal{C}\left(\lambda k^{\prime} . J^{t p}\right) \text { in } N^{t p}\right) \\
\rightarrow_{\text {Sub } b_{v}^{\text {lift }}} & \mathcal{C}\left(\lambda q . J^{t p}\left[\text { let }(f, t p)=\square \text { in } q\left(f N^{t p}\right) / k^{\prime}\right]\right) \\
= & \mathcal{C}\left(\lambda q . J^{t p}\left[(q(\square N))^{+} / k^{\prime}\right]\right) \\
\rightarrow & P \\
\leftarrow_{\mathcal{C}_{\text {idem }}} & \left(\mathcal{C}\left(\lambda q . J\left[q(\square N) / k^{\prime}\right]\right)\right)^{t p} \quad \text { (by Lemma 20(iii)) }
\end{array}
$$

REMARK 22. In trying to find a better simulation of $\lambda_{\widehat{\mathcal{C t p}}}$, we have investigated alternative subtraction rules. In particular, the exact form to give to the elimination rule is not obvious in natural deduction (compared to its definition in sequent calculus (Curien and Herbelin, 2000)). For example, the rule:

$$
\frac{\Gamma \vdash M: A-B \quad \Gamma, x: A, k: B \rightarrow \Perp \vdash M^{\prime}: C}{\Gamma \vdash \operatorname{let}(x, k)=M \text { in } M^{\prime}: C}
$$

that has been considered in a previous work (Ariola et al., 2004) seems to be equally worthwhile. This however complicates the translation of $\widehat{t p} M$, which becomes (tp (join $\left.M^{t p}\right)$ ), where join $M$ is now defined as let $(x, k)=M$ in $T h k x$. Due to the outer occurrence of $t p$, the translation is not stable under reduction. This introduces the need of reasoning up to the name of this extra continuation variable. Another solution is to reason in full classical logic with subtraction (i.e. $\lambda_{\mathcal{c}}^{-\perp}$ ) instead of minimal classical logic (i.e. $\left.\lambda_{\mathcal{C}}^{-}\right)$and to define $\widehat{t p} M$ as $\operatorname{tp}_{\perp}\left(\right.$ join $\left.M^{t p}\right)$, where $\mathrm{tp}_{\perp}$ witnesses the elimination rule of $\perp$ (see Intermezzo 16). We believe that a true simulation of the reduction would have been obtained with this approach.

As a corollary, we get the following result.

PROPOSITION 23. If $M={ }_{\lambda_{\widehat{C} \widehat{t p}}} N$ then $M^{t p}={ }_{\lambda_{\mathcal{C}}} N^{t p}$.

As an emphasis of how the translation works, the following example shows how to capture dynamic substitution of $\lambda_{\widehat{\mathcal{C t p}}}$.

EXAMPLE 24. Consider Example 6 that was used to illustrate the capture of $\widehat{t p}$ during substitution:

$$
\begin{aligned}
& (\lambda x \cdot(\lambda y \cdot \mathcal{C}(\lambda \widehat{t p} \cdot \widehat{t p}(x y))))\left(\lambda_{-} \cdot \mathcal{C}\left(\lambda_{-} \cdot \widehat{t p} y\right)\right) \\
\rightarrow & \lambda y^{\prime} \cdot \mathcal{C}\left(\lambda \widehat{t p} \cdot \widehat{t p}\left(\left(\lambda_{-} \cdot \mathcal{C}\left(\lambda_{-} . \widehat{t p} y\right)\right) y^{\prime}\right)\right)
\end{aligned}
$$


The embedding of the left-hand side is as follows:

$$
\begin{array}{ll}
\left((\lambda x \cdot(\lambda y \cdot \mathcal{C}(\lambda \widehat{t p} \cdot \widehat{t p}(x y))))\left(\lambda_{-} \cdot \mathcal{C}\left(\lambda_{-} \cdot \widehat{t p} y\right)\right)\right)^{t p} & = \\
(\lambda x \cdot(\lambda y \cdot \mathcal{C}(\lambda \widehat{t p} \cdot \widehat{t p}(x y))))^{+}\left(\lambda_{-} \cdot \mathcal{C}\left(\lambda_{-} \cdot \widehat{t p} y\right)\right)^{t p} & = \\
\left(\lambda\left(x, t p_{1}\right) \cdot(\lambda y \cdot \mathcal{C}(\lambda \widehat{t p} \cdot \widehat{t p}(x y)))^{t p_{1}}\right)\left(\left(\lambda_{-} \cdot \mathcal{C}\left(\lambda_{-} \cdot \widehat{t p} y\right)\right)^{+}, t p\right) & = \\
\left(\left(\lambda\left(x, t p_{1}\right) \cdot\left(\left(\lambda\left(y, t p_{3}\right) \cdot \mathcal{C}(\lambda \widehat{t p} \cdot \widehat{t p}(x y))^{t p_{3}}\right), \lambda x \cdot t p_{1} x\right)\right)\right. & = \\
\left.\left(\left(\lambda_{-} \cdot \mathcal{C}\left(\lambda_{-} \cdot \widehat{t p} y\right)\right)^{+}, t p\right)\right) & \left(\left(\lambda\left(x, t p_{1}\right) \cdot\left(\lambda\left(y, t p_{3}\right) \cdot\left(\mathcal{C}\left(\lambda t p_{4} \cdot \text { join }\left(x\left(y, t p_{4}\right)\right)\right), \lambda x \cdot t p_{3} x\right), \lambda x \cdot t p_{1} x\right)\right)\right. \\
\left.\left(\left(\lambda\left(-, t p_{2}\right) \cdot \mathcal{C}\left(\lambda_{-} \text {join }\left(y, \lambda x \cdot t p_{2} x\right)\right)\right), t p\right)\right) &
\end{array}
$$

This term reduces to:

$$
\begin{gathered}
\left(\lambda ( y ^ { \prime } , t p _ { 3 } ) \cdot \left(\mathcal { C } \left(\lambda t p _ { 4 } . \text { join } \left(\left(\lambda\left(-, t p_{2}\right) \cdot \mathcal{C}\left(\lambda_{-} . \text {join }\left(y, \lambda x . t p_{2} x\right)\right)\right)\right.\right.\right.\right. \\
\left.\left.\left.\left.\left(y^{\prime}, t p_{4}\right)\right)\right), \lambda x . t p_{3} x\right), t p\right)
\end{gathered}
$$

which is the embedding of the right-hand side.

The theory $\lambda_{\mathcal{C}}^{-}$is richer than $\lambda_{\mathcal{C} \widehat{c t p}}$ or $\lambda_{\mathcal{C} \# \text { tp }}$, so that the translation is not complete.

EXAMPLE 25. Consider the $\lambda_{\mathcal{C} \# \text { tp }}$ term $y(\#(\operatorname{tp} x))$ which is mapped into the $\lambda_{\mathcal{C}}^{-}$term $y(\mathcal{C}(\lambda t p$. $\operatorname{join}(x, t p), t p)$, which reduces as follows:

$$
\begin{aligned}
& y(\mathcal{C}(\lambda t p . \operatorname{join}(x, t p)), t p) \stackrel{\text { Subuase }}{\longrightarrow} y((\mathcal{C}(\lambda t p . t p x), t p)) \\
& \stackrel{\mathcal{C}_{\text {lift }}}{\longrightarrow} y(\mathcal{C}(\lambda t p \cdot t p(x, t p))) \\
& \stackrel{\mathcal{C}_{\text {lift }}}{\longrightarrow} \mathcal{C}(\lambda t p . t p(y(x, t p)))
\end{aligned}
$$

The lift reductions move the \# while maintaining the proper references to the top-level continuation. This move has no counterpart in either $\lambda_{\mathcal{C} \# \text { tp }}$ or $\lambda_{\mathcal{C} \widehat{t p}}$. In $\lambda_{\mathcal{C} \widehat{c t p}}$ such a lifting would cause the dynamically-bound top-level continuation $\widehat{t p}$ to be incorrectly captured; in $\lambda_{\mathcal{C}}^{-}$, the toplevel continuation is statically-bound so that it is enough to rely on $\alpha$-conversion to have a safe lifting rule for \#. Pulling the lifting rule for \# back from $\lambda_{\mathcal{C}}^{-}$to $\lambda_{\widehat{\mathcal{c t p}}}$ is non trivial, if even possible.

\subsection{Which TYPE SYSTEMS ENSURE TERMINATION?}

We are now able to show that the type systems with effects ensure strong normalisation of the underlying $\lambda$-calculus while the type system with fixed type does not.

\section{PROPOSITION 26.}

(i) If $\Gamma ; U \vdash M: A ; T$ in $\underset{\Lambda_{\widehat{C} t p}}{\rightarrow}$ eff or $\Gamma \vdash M: A ; T$ in $\underset{\Lambda_{\widehat{C} t p}^{\rightarrow}}{\rightarrow \text { effeq }}$ then $M$ is strongly normalising. 
(ii) If $\Gamma \vdash M: A ; T$ in $\underset{\widehat{\mathcal{C} t p}}{\rightarrow \text { fixed }}$ and $T$ is atomic then $M$ is strongly normalising.

Proof. The result follows from Propositions 12, 17, 18, 21 and the fact that $\mathcal{C}_{\text {idem }}$ commutes with the other reductions in the following way: if $M \rightarrow N$ and $M \rightarrow \mathcal{C}_{\text {idem }} M^{\prime}$ then there is $N^{\prime}$ such that $M^{\prime} \rightarrow N^{\prime}$ and $N \rightarrow \mathcal{C}_{\text {idem }} N^{\prime}$, unless $M \rightarrow \mathcal{C}_{\text {elim }} N=M^{\prime}$. But since $\mathcal{C}_{\text {elim }}$ cannot be applied infinitely many times in a row, any infinite reduction sequence

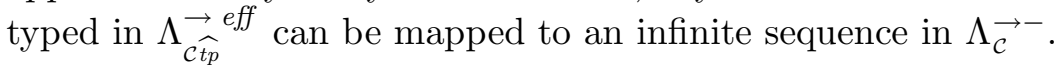

Strong normalisation does not generally hold in $\Lambda_{\widehat{C} \widehat{t p}}^{\rightarrow}$ fixed if the toplevel type is not atomic. Indeed, if $T$ is non atomic then we have to add the annotation $T$ on the arrows of $T$ itself. This requires a definition by fixpoint and the resulting recursive type can be used to type a fixpoint combinator as shown in Example 8.

\section{CPS Semantics}

To complete our investigation, we present a CPS semantics for $\lambda_{\mathcal{C}}^{-}$and a corresponding double-negation translation for $\Lambda_{\mathcal{c}}-$.

\subsection{Target CPS Calculus}

Before presenting the CPS translation from $\lambda_{\mathcal{C}}^{-}$we first introduce the target language of the translation. This target language $\lambda^{\wedge}$ is an ordinary call-by-name $\lambda$-calculus with pairs. The semantics of $\lambda^{\wedge}$ is given using the following two rules:

$$
\begin{array}{ll}
\beta:(\lambda x \cdot M) N & \rightarrow M[N / x] \\
\wedge: \text { let }(x, y)=(M, N) \text { in } M^{\prime} \rightarrow M^{\prime}[N / y ; M / x]
\end{array}
$$

We also use the following abbreviation:

$$
\lambda(x, y) \cdot M \triangleq \lambda z . \text { let }(x, y)=z \text { in } M
$$

The type system of $\lambda^{\wedge}$, named $\Lambda^{\wedge}$, is an ordinary natural deduction system equipped with tensorial conjunction. This is a conjunction whose elimination rule extracts both components of the pair simultaneously, similar to what happens for the tensor product of linear logic. The tensorial conjunction will be used to model subtractions in $\lambda_{\mathcal{C}}^{-}$; its introduction and elimination rules are as follows: 


$\begin{array}{ll}b^{*} & =b \\ (A \rightarrow B)^{*} & =\neg B^{*} \rightarrow \neg A^{*} \\ (A-B)^{*} & =\neg B^{*} \wedge A^{*} \\ & =\cdot \\ (\cdot)^{*} & =\Gamma^{*}, x: A^{*} \\ (\Gamma, x: A)^{*} & \\ (\Gamma, k: A \rightarrow \Perp)^{*} & =\Gamma^{*}, k: \neg A^{*} \\ & \\ (\Gamma \vdash M: A)^{k} & =\Gamma^{*}, k: \neg A^{*} \vdash \llbracket M \rrbracket k: \perp \\ (\Gamma \vdash J: \Perp)^{*} & =\Gamma^{*} \vdash \llbracket J \rrbracket: \perp\end{array}$

Figure 22. Fischer-style call-by-value $\neg \neg$-translation on $\Lambda_{\mathcal{C}}-$

$$
\begin{gathered}
\frac{\Gamma \vdash M: A \quad \Gamma \vdash N: B}{\Gamma \vdash(M, N): A \wedge B} \wedge_{i} \\
\frac{\Gamma \vdash M: A \wedge B \quad \Gamma, x: A, y: B \vdash M^{\prime}: C}{\Gamma \vdash \operatorname{let}(x, y)=M \text { in } M^{\prime}: C} \wedge_{e}
\end{gathered}
$$

We assume the existence in $\Lambda^{\wedge}$ of a distinguished formula $\perp$ to which no inference rule is associated. We write $\neg A$ as an abbreviation of $A \rightarrow \perp$.

\subsection{CPS Translation of $\lambda_{\mathcal{C}}^{-}$}

The CPS translation maps the types, judgements, and terms of $\lambda_{\mathcal{C}}^{-}$to $\lambda^{\wedge}$. The translation we present extends the call-by-value CPS translation of Fischer $(1972,1993)$. We give the translation in Figure 22 for the types and judgements and in Figure 23 for the terms and the contexts. We have:

PROPOSITION 27. The CPS translation is sound with respect to typing:

$$
\begin{aligned}
& - \text { If } \Gamma \vdash M: A \text { in } \Lambda_{\mathcal{c}}^{-} \text {then }(\Gamma \vdash M: A)^{k} \text { in } \Lambda^{\wedge} \\
& - \text { If } \Gamma \vdash J: \Perp \text { in } \Lambda_{\mathcal{c}}^{-} \text {then }(\Gamma \vdash J: \Perp)^{*} \text { in } \Lambda^{\wedge} .
\end{aligned}
$$

Before proving the soundness of the translation with respect to reductions, we first state a few useful properties.

LEMMA 28. 


\begin{tabular}{|c|c|}
\hline $\begin{array}{l}x^{+} \\
(\lambda x . M)^{+} \\
\left(V, \lambda x . J_{c}[E[x]]\right)^{+}\end{array}$ & $\begin{array}{l}=x \\
=\lambda k^{\prime} \cdot \lambda x \cdot \llbracket M \rrbracket k^{\prime} \\
=\left(\llbracket E \rrbracket \llbracket J_{c} \rrbracket, V^{+}\right)\end{array}$ \\
\hline $\begin{array}{l}\llbracket V \rrbracket k \\
\llbracket V \quad N \rrbracket k \\
\llbracket M \quad N \rrbracket k\end{array}$ & $\begin{array}{l}=k V^{+} \\
=\llbracket N \rrbracket\left(V^{+} k\right) \\
=\llbracket M \rrbracket(\lambda f \cdot \llbracket N \rrbracket(f k)) \quad(M \text { not value })\end{array}$ \\
\hline $\begin{array}{l}\llbracket \mathcal{C}\left(\lambda k^{\prime} . J\right) \rrbracket k \\
\llbracket\left(M, \lambda x . J_{c}[E[x]]\right) \rrbracket k\end{array}$ & $\begin{array}{l}=\llbracket J \rrbracket\left[k / k^{\prime}\right] \\
=\llbracket M \rrbracket\left(\lambda v \cdot k\left(\llbracket E \rrbracket \llbracket J_{c} \rrbracket, v\right)\right) \quad(M \text { not value })\end{array}$ \\
\hline $\begin{array}{l}\llbracket \text { let }(x, k)=M \text { in } J \rrbracket \\
\llbracket k M \rrbracket\end{array}$ & $\begin{array}{l}=\llbracket M \rrbracket(\lambda(k, x) \cdot \llbracket J \rrbracket) \\
=\llbracket M \rrbracket k\end{array}$ \\
\hline $\begin{array}{l}\llbracket \square N \rrbracket k \\
\llbracket V \square \rrbracket k \\
\llbracket\left(\square, \lambda x . J_{c}[E[x]]\right) \rrbracket k\end{array}$ & $\begin{array}{l}=\lambda f \cdot \llbracket N \rrbracket(f k) \\
=V^{+} k \\
=\lambda v \cdot k\left(\llbracket E \rrbracket \llbracket J_{c} \rrbracket, v\right)\end{array}$ \\
\hline $\begin{array}{l}\llbracket E[F] \rrbracket k \\
\llbracket \square \rrbracket k\end{array}$ & $\begin{array}{l}=\llbracket F \rrbracket(\llbracket E \rrbracket k) \\
=k\end{array}$ \\
\hline $\begin{array}{l}\llbracket k \square \rrbracket \\
\llbracket \text { let }(x, k)=\square \text { in } J \rrbracket\end{array}$ & $\begin{array}{l}=k \\
=\lambda(k, x) \cdot \llbracket J \rrbracket\end{array}$ \\
\hline
\end{tabular}

Figure 23. Fischer-style call-by-value CPS translation of $\lambda_{\mathcal{C}}^{-}$

$\begin{array}{llll}\text { (i) } & \llbracket M \rrbracket(\llbracket F \rrbracket k) & =\llbracket F[M] \rrbracket k & \text { if } M \text { not a value } \\ \text { (ii) } & \llbracket V \rrbracket(\llbracket F \rrbracket k) & \rightarrow \llbracket F[V] \rrbracket k \\ \text { (iii) } & \llbracket M \rrbracket \llbracket J_{c} \rrbracket & =\llbracket J_{c}[M] \rrbracket \\ \text { (iv) } & \llbracket M[V / x] \rrbracket k & =(\llbracket M \rrbracket k)\left[V^{+} / x\right] \\ \text { (v) } & \llbracket J[V / x] \rrbracket & =\llbracket J \rrbracket\left[V^{+} / x\right] \\ \text { (vi) } & \llbracket F[E] \rrbracket k & =\llbracket E \rrbracket(\llbracket F \rrbracket k) \\ \text { (vii) } & \llbracket J \rrbracket\left[\llbracket F \rrbracket k^{\prime} / k\right] & \rightarrow \llbracket J\left[k^{\prime} F / k\right] \rrbracket \\ \text { (viii) } & \llbracket F[\mathcal{C}(\lambda k . J)] \rrbracket k^{\prime} & \rightarrow \llbracket J\left[k^{\prime} F / k\right] \rrbracket \\ \text { (ix) } & \llbracket J \rrbracket\left[\llbracket J_{c} \rrbracket / k\right] & =\llbracket J\left[J_{c} / k\right] \rrbracket \\ \text { (x) } & \llbracket J_{c}[\mathcal{C}(\lambda k . J)] \rrbracket & =\llbracket J\left[J_{c} / k\right] \rrbracket\end{array}$

Proof. Items $(i),(i i i),(i v)$ and $(v)$ can be easily checked. Item (ii) introduces a reduction for the contexts $\square N$ and $\left(\lambda x . J_{c}[E[x]], \square\right)$. Items $(v i)$ is by induction on the length of $E$. For item (vii), many cases can occur. If $k$ occurs at the head of the second component of a subtractive term, the substitution leaves the term unchanged because of item (vi). If $k$ occurs elsewhere, then it may be applied to a value or not and either $(i)$ or $(i i)$ applies. For item (viii), the result follows from $(i)$ and 
(vii). Item $(i x)$ follows because $k$ is substitutive when it occurs at the head of the second component of a subtractive term and by item (iii) otherwise. For item $(x)$, the result follows from $(i i i)$ and $(i x)$.

PROPOSITION 29. The CPS translation is sound with respect to the $\lambda_{\mathcal{C}}^{-}$semantics:

(i) If $M \rightarrow M^{\prime}$ in $\lambda_{\mathcal{C}}^{-}$then $\llbracket M \rrbracket k=\beta, \wedge \llbracket M^{\prime} \rrbracket k$ in $\lambda^{\wedge}$;

(ii) If $J \rightarrow J^{\prime}$ in $\lambda_{\mathcal{c}}^{-}$then $\llbracket J \rrbracket=\beta, \wedge \llbracket J^{\prime} \rrbracket$ in $\lambda^{\wedge}$;

Proof. We consider a few of the cases:

- $S u b_{v}^{\text {lift }}$ : From Lemma 28(x), we directly have:

$\llbracket$ let $(x, q)=\mathcal{C}\left(\lambda k . J^{\prime}\right)$ in $J \rrbracket=\llbracket J^{\prime}[$ let $(x, q)=\square$ in $J / k] \rrbracket$.

- $S u b_{v}^{\text {base }}$ : The left-hand side is:

$$
\begin{aligned}
\llbracket \text { let }(x, q)=\left(V, k^{\prime}\right) \text { in } J \rrbracket & =\llbracket\left(V, k^{\prime}\right) \rrbracket(\lambda(q, x) . \llbracket J \rrbracket) \\
& \rightarrow \beta_{v}\left(\text { let }(q, x)=\left(k^{\prime}, V^{+}\right) \text {in } \llbracket J \rrbracket\right. \\
& \rightarrow \wedge \llbracket J \rrbracket\left[k^{\prime} / q ; V^{+} / x\right]
\end{aligned}
$$

The right-hand side is $\llbracket J\left[k^{\prime} / q ; V / x\right] \rrbracket$ and the result follows from Lemma $28(v, x)$.

- $S u b_{v}^{\text {step }}$ : The left-hand side is:

$$
\begin{aligned}
& \llbracket \text { let }\left(x, k^{\prime}\right)=\left(V, \lambda x . J_{c}[E[F[x]]]\right) \text { in } J \rrbracket= \\
& \left(\lambda k . k\left(\llbracket F \rrbracket\left(\llbracket E \rrbracket \llbracket J_{c} \rrbracket\right), V^{+}\right)\right)\left(\lambda\left(k^{\prime}, x\right) . \llbracket J \rrbracket\right) \rightarrow_{\beta_{v}} \\
& \text { let }\left(k^{\prime}, x\right)=\left(\llbracket F \rrbracket\left(\llbracket E \rrbracket \llbracket J_{c} \rrbracket\right), V^{+}\right) \text {in } \llbracket J \rrbracket \rightarrow \wedge \\
& \llbracket J \rrbracket\left[\llbracket F \rrbracket\left(\llbracket E \rrbracket \llbracket J_{c} \rrbracket\right) / k^{\prime} ; V^{+} / x\right]= \\
& \llbracket J \rrbracket\left[\llbracket F \rrbracket k^{\prime \prime} / k^{\prime}\right]\left[\llbracket E \rrbracket \llbracket J_{c} \rrbracket / k^{\prime \prime} ; V^{+} / x\right] \quad \text { for } k^{\prime \prime} \text { fresh }
\end{aligned}
$$

For the right-hand side we have:

$$
\begin{aligned}
& \text { 【let }\left(x, k^{\prime}\right)=\left(V, \lambda x . J_{c}[E[x]]\right) \text { in } J\left[k^{\prime} F / k^{\prime}\right] \rrbracket \rightarrow \beta_{v} \\
& \text { let }\left(k^{\prime}, x\right)=\left(\llbracket E \rrbracket \llbracket J_{c} \rrbracket, V^{+}\right) \text {in } \llbracket J\left[k^{\prime} F / k^{\prime}\right] \rrbracket \rightarrow \wedge \\
& \left(\llbracket J\left[k^{\prime} F / k^{\prime}\right] \rrbracket\right)\left[\llbracket E \rrbracket \llbracket J_{c} \rrbracket / k^{\prime} ; V^{+} / x\right]
\end{aligned}
$$

We conclude by Lemma 28(vii). 


$\begin{array}{ll}b^{*} & =b \\ \left(A_{U \rightarrow T} B\right)^{*} & =\neg\left(\neg U^{*} \wedge B^{*}\right) \rightarrow \neg\left(\neg T^{*} \wedge A^{*}\right) \\ (A \rightarrow U \Perp)^{*} & =\neg\left(\neg U^{*} \wedge A^{*}\right) \\ & \\ (\Gamma ; B \vdash M: A ; C)_{k}^{t p} & =\Gamma^{*}, t p: \neg C^{*}, k: \neg\left(\neg B^{*} \wedge A^{*}\right) \vdash \llbracket M \rrbracket k t p: \perp \\ (\Gamma \vdash J: \Perp ; C)^{t p} & =\Gamma^{*}, t p: \neg C^{*} \vdash \llbracket J \rrbracket t p: \perp\end{array}$

Figure 24. Derived call-by-value $\neg \neg$-state-transformation on $\Lambda_{\mathcal{C} \text { tp }}$ eff

$$
\begin{array}{ll}
x^{+} & =x \\
(\lambda x \cdot M)^{+} & =\lambda k \cdot \lambda(t p, x) \cdot \llbracket M \rrbracket k t p \\
& =k\left(t p, V^{+}\right) \\
\llbracket V \rrbracket k t p & =\llbracket N \rrbracket\left(V^{+} k\right) t p \\
\llbracket V N \rrbracket k t p & =\llbracket M \rrbracket\left(\lambda\left(t p^{\prime}, f\right) . \llbracket N \rrbracket(f k) t p^{\prime}\right) t p \quad M \text { not a value } \\
\llbracket M N \rrbracket k t p & \\
\llbracket \mathcal{C}\left(\lambda k^{\prime} . J\right) \rrbracket k t p & =(\llbracket J \rrbracket t p)\left[k / k^{\prime}\right] \\
\llbracket \# J \rrbracket k t p & =\llbracket J \rrbracket \lambda v \cdot k(t p, v) \\
\llbracket t p M \rrbracket t p & =\llbracket M \rrbracket(\lambda(k, x) \cdot k x) t p \\
\llbracket k M \rrbracket t p & =\llbracket M \rrbracket k t p
\end{array}
$$

Figure 25. Derived call-by-value CPS translation of $\lambda_{\mathcal{C} \# \text { tp }}$

\subsection{Induced CPS TRANSLation of $\lambda_{\mathcal{C} \# \text { tp }}$}

As $\lambda_{\mathcal{C} \# \text { tp }}$ and $\lambda_{\mathcal{C} \widehat{c p}}$ are isomorphic, we can compose the embeddings from $\lambda_{\mathcal{C} \widehat{t p}}$ to $\lambda_{\mathcal{C}}^{-}$with the CPS translation from $\lambda_{\mathcal{c}}^{-}$to $\lambda^{\wedge}$ to produce a CPS translation for $\lambda_{\mathcal{C} \# \text { tp }}$. The result is given in Figures 24 and 25 (obvious redexes have been contracted), where $\Lambda_{\mathcal{C t p}}$ eff stands for the type-andeffect system of $\lambda_{\mathcal{C} \# \text { tp }}$. This is obtained from the system of Figure 13 by replacing the $R A A^{\widehat{t p}}$ and $\rightarrow \rightarrow_{e}^{\widehat{t p}}$ with the following typing rules:

$$
\frac{\Gamma \vdash J: \Perp ; A}{\Gamma ; T \vdash \# J: A ; T} \quad \frac{\Gamma ; U \vdash M: U ; T}{\Gamma \vdash \operatorname{tp} M: \Perp ; T}
$$

The $\lambda_{\mathcal{C} \# \text { tp }}$ CPS translation shows how such a translation for delimited continuations can be decomposed into a store-passing transformation followed by a standard CPS transformation into the pure $\lambda$-calculus. The first transformation deals with the control delimiter; the second transformation deals with the control operator. By putting together Propositions 7, 23 and 29, one then concludes that the CPS semantics preserves the notions of convertibility of $\lambda_{\mathcal{C} \# \mathrm{tp}}$.

PROPOSITION 30. Given $\lambda_{\mathcal{C} \# \text { tp }}$ terms $M$ and $N$. If $M=N$ in $\lambda_{\mathcal{C} \# \text { tp }}$ then $\llbracket M \rrbracket k t p=\beta, \wedge \llbracket N \rrbracket k t p$. 


\subsection{The Operators shift AND reset}

Based on the embeddings of shift and reset given in Remark 4, their translations in $\lambda^{\wedge}$ are:

$$
\begin{array}{ll}
\llbracket\langle M\rangle \rrbracket k t p & =\llbracket M \rrbracket I^{\prime}(\lambda v . k(t p, v)) \\
\llbracket \mathcal{S}(\lambda q . M) \rrbracket k t p & =\llbracket M \rrbracket[\text { reify } S k / q] I^{\prime} t p
\end{array}
$$

where $I^{\prime}$

$$
=\lambda(k, x) \cdot k x
$$

$$
\text { reifyS } k=\lambda k^{\prime} \cdot \lambda(t p, x) \cdot k\left(\lambda y \cdot k^{\prime}(t p, y), x\right)
$$

which correspond, up to currying and $\eta$-conversion, to the definition of shift and reset in the original paper by Danvy and Filinski (1989).

Let $\lambda_{\mathcal{C} \# \text { tp }}^{\prime}$ be the subset of $\lambda_{\mathcal{C} \# \text { tp }}$ without continuation variables and where every occurrence of tp and $\mathcal{C}$ only occurs as part of the definitions of $\mathcal{S}$ and $\left\langle_{-}\right\rangle$, but still equipped jwith the same equational theory as $\lambda_{\mathcal{C} \# \text { tp }}$. What we have shown is that the following diagram commutes:

\section{THEOREM 31.}

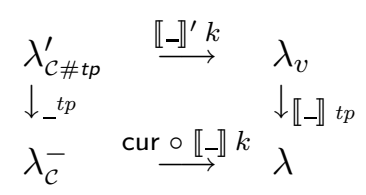

where

$-\lambda_{v}$ is Plotkin's call-by-value $\lambda$-calculus and $\lambda$ is usual $\lambda$-calculus,

- cur is the currying combinator,

- $\llbracket \rrbracket \rrbracket^{\prime} k$ is the same as $\llbracket-\rrbracket k$ from Figure 23 but extended with the clauses

$$
\begin{aligned}
\llbracket \# J \rrbracket^{\prime} k & =k \llbracket J \rrbracket^{\prime} \\
\llbracket t p M \rrbracket^{\prime} & =\llbracket M \rrbracket^{\prime} \lambda x . x
\end{aligned}
$$

so that the “macros" $\llbracket\langle M\rangle \rrbracket^{\prime} k$ and $\llbracket \mathcal{S}(\lambda q . M) \rrbracket^{\prime} k$ are translated as in (Danvy and Filinski, 1990),

- 【-』tp is as in Figure 23 but written with the name tp in place of $k$, so that the up and right sides of the square correspond to the two-steps CPS translation from the syntax of $\lambda_{\mathcal{C} \# t p}^{\prime}$ to $\lambda$ (Danvy and Filinski, 1990). 


\subsection{Completeness of the CPS Translation}

Thanks to Kameyama and Hasegawa (2003) axiomatisation of $\lambda_{\mathcal{C} \# \text { tp }}^{\prime}$, we can give a proof of completeness of our translation. Let $=_{\lambda_{\mathcal{C} \# \text { tp }}^{\prime}}$ be the extension of $={ }_{\lambda_{\mathcal{C} \# \mathrm{tp}}}$ obtained by adding the following reductions:

$$
\begin{aligned}
& \#_{\text {idem }}: \operatorname{tp}(\# J) \quad \rightarrow^{\prime} J \\
& \#_{\text {tail }}: \quad \#(l((\lambda x . M)(\# J))) \rightarrow^{\prime}(\lambda x . \#(l M))(\# J) \\
& \mathcal{C}_{\text {tail }}: \quad(\lambda x . \mathcal{C}(\lambda k . l M)) N \quad \rightarrow^{\prime} \mathcal{C}(\lambda k . l((\lambda x . M) N)) \\
& \eta_{v}: \quad \lambda x .\left(\begin{array}{lll} 
& x) & \rightarrow
\end{array} \quad \text { where } x \notin F V(V)\right. \\
& \beta_{\Omega}: \quad(\lambda x . E[x]) M \quad \rightarrow^{\prime} E[M] \quad \text { where } x \notin F V(E)
\end{aligned}
$$

where $l$ is either $k$ or tp, $F$ is either $V \square$ or $\square N$ and $E$ is either or $E[F]$. The following equations (respectively consequences of $\beta_{\Omega}$ and $\#_{\text {idem }}$ conjugated with $\mathcal{C}_{\text {idem }}$ and $\mathcal{C}_{\text {tail }}$, of $\mathcal{C}_{\text {tail }}, \mathcal{C}_{\text {idem }}$ and $\beta_{\Omega}$, and of $\beta_{\Omega}$ and $\beta_{v}$ ) will be useful:

$$
\begin{aligned}
& \#_{\text {let }}: \quad \operatorname{tp}((\lambda x . \#(k x)) M)=_{\lambda_{\mathcal{C} \# \mathrm{tp}}}^{\prime} k M \\
& T_{\text {let }}: \operatorname{tp}((\lambda x \cdot \text { Th }(k x)) M)=_{\lambda_{\mathcal{C} \# \mathrm{tp}}}^{\prime} k M \\
& \text { let }_{\text {lift }}: F[(\lambda x . N) M] \quad=_{\lambda_{\mathcal{C \# t p}}}^{\prime}(\lambda x . F[N]) M \text {. }
\end{aligned}
$$

Kameyama and Hasegawa's axioms of $\lambda_{\mathcal{C} \# \text { tp }}^{\prime}$ are listed below. Since $\mathcal{S}$ is a stand-alone constant in Kameyama and Hasegawa (2003), we need to slightly adapt their axiomatic ${ }^{1}$ :

$$
\begin{aligned}
& \beta_{v}: \quad(\lambda x . M) V \quad={ }_{K H} \quad M[V / x] \\
& \mathcal{S} \text {-reset }: \quad\langle E[\mathcal{S}(\lambda k . M)]\rangle \quad={ }_{K H} \quad\langle(\lambda k . M) \lambda x \cdot\langle E[x]\rangle\rangle \\
& \mathcal{S} \text {-elim : } \quad \mathcal{S}(\lambda k . k M) \quad={ }_{K H} \quad M \quad \text { where } k \notin F V(M) \\
& \mathcal{S} \text {-tail : } \quad(\lambda x . \mathcal{S}(\lambda k . M)) N={ }_{K H} \mathcal{S}(\lambda k .(\lambda x . M) N) \\
& \text { reset-value: }\langle V\rangle \quad=K H \quad V \\
& \text { reset-lift : } \quad\langle(\lambda x . M)\langle N\rangle\rangle \quad{ }_{K H}(\lambda x .\langle M\rangle)\langle N\rangle \\
& \text { reset-S }: \mathcal{S}(\lambda k .\langle M\rangle) \quad=K H \quad \mathcal{S}(\lambda k . M) \\
& \eta_{v}: \quad \lambda x \cdot(V x) \quad{ }_{K H} \quad V \quad \text { where } x \notin F V(V) \\
& \beta_{\Omega}: \quad(\lambda x . E[x]) M \quad=K H \quad E[M] \quad \text { where } x \notin F V(E)
\end{aligned}
$$

On $\lambda_{\mathcal{C} \# \text { tp }}^{\prime}$, Kameyama and Hasegawa's axiomatisation is derivable from the equational theory $={ }_{\lambda_{\mathcal{C} \# \mathrm{tp}}}^{\prime}$ :

\footnotetext{
1 The original system has the rule $\mathcal{S}$-reset : $\langle E[\mathcal{S} M]\rangle=_{K H}\langle M \lambda x .\langle E[x]\rangle\rangle$ and no rule $\mathcal{S}$-tail. The full strength of the original $\mathcal{S}$-reset is not expressible in $\lambda_{\mathcal{C} \# \text { tp }}^{\prime}$ since $\mathcal{S}$ in there is constrained to appear with the form $\mathcal{S}(\lambda k . M)$. The original $\mathcal{S}$-reset implies $\mathcal{S}_{\eta}: \mathcal{S} M=\mathcal{S}(\lambda k . M k)$, so, obviously, it is equivalent to the combination of $\mathcal{S}_{\eta}$ and of the modified $\mathcal{S}$-reset. Thanks to $\beta_{\Omega}$ and $\eta_{v}, \mathcal{S}_{\eta}$ is provably equivalent to $\mathcal{S}$-tail, hence the modified system is equivalent to the original one on $\lambda_{\mathcal{C} \# \text { tp }}^{\prime}$ We leave open the question whether $\mathcal{S}$-tail is not redundant in the modified system.
} 
PROPOSITION 32. Let $M$ and $N$ be in $\lambda_{\mathcal{C} \# t p}^{\prime}$. If $M=_{K H} N$ then $M={ }_{\lambda_{\mathcal{C} \# t p}^{\prime}}^{\prime} N$.

Proof. The rules $\beta_{v}, \eta_{v}, \beta_{\Omega}$ and reset-value are also in $=_{\lambda_{\mathcal{C \# t}}^{\prime}}^{\prime} ; \mathcal{S}$-reset is a combination of $\mathcal{C}_{L}, \mathcal{C}_{R}$ and $\mathcal{C}_{\text {idem }} ; \mathcal{S}$-elim is a consequence of $\mathcal{C}_{\text {elim }}$ using first $\beta_{v}, \#_{\text {let }}$ and $\#_{\text {idem }} ; \mathcal{S}$-tail is a consequence of $\mathcal{C}_{\text {tail }}$ and let lift; reset-lift derives from $\#_{\text {tail }}$ for $l$ being tp; finally, reset- $\mathcal{S}$ derives from $\#_{\text {idem }}$ conjugated with $\beta_{v}$.

Now, since Kameyama and Hasegawa's axiomatisation is complete with respect to the CPS translation ${ }^{2}$, we get $(\eta \wedge$ is the equation $M=$ $($ let $(x, y)=M$ in $x$, let $(x, y)=M$ in $y))$ :

COROLLARY 33. Given $\lambda_{\mathcal{C} \# \text { tp }}^{\prime}$ terms $M$ and $N$, if $\llbracket M \rrbracket k t p={ }_{\beta, \eta, \wedge, \eta \wedge}$ $\llbracket N \rrbracket k$ tp then $M={ }_{\lambda_{\mathcal{C} \# t p}^{\prime}}^{\prime} N$.

We then show that any term $M$ in $\lambda_{\mathcal{C} \# \text { tp }}$ has a representative in $\lambda_{\mathcal{C} \# \text { tp }}^{\prime}$ that behaves the same with respect to $={ }_{\lambda_{\mathcal{C} \# \text { tp }}^{\prime}}$. Let $\lambda_{\mathcal{C} \# \text { tp }}^{-}$be the subset of $\lambda_{\mathcal{C} \# \text { tp }}$ with no free continuation variables. The following embedding, that relies on the definition of $\mathcal{S}$ and \langle\rangle in Remark 4, is a map from $\lambda_{\mathcal{C} \# \text { tp }}^{-}$to $\lambda_{\mathcal{C} \# \text { tp }}^{\prime}$ :

$$
\begin{array}{llll}
x^{\bullet} & =x & \\
(\lambda x . M)^{\bullet} & =\lambda x . M^{\bullet} & & (\operatorname{tp} M)^{\bullet}=M^{\bullet} \\
(M N)^{\bullet} & =M^{\bullet} N^{\bullet} & & (k M)^{\bullet}=k M^{\bullet} \\
(\mathcal{C}(\lambda k . J))^{\bullet} & =\mathcal{S}\left(\lambda k . J^{\bullet}\right) & & \\
(\# J)^{\bullet} & =\left\langle J^{\bullet}\right\rangle & &
\end{array}
$$

Notice that $(k M)^{\bullet}$, when in position of subterm, is necessarily surrounded by tp. Thanks to the following equation:

$$
\begin{array}{rll}
(\mathcal{C}(\lambda k . J))^{\bullet} & = & \mathcal{C}\left(\lambda k \cdot \operatorname{tp}\left(\left(\lambda k . J^{\bullet}\right) \lambda x \cdot \#(k x)\right)\right) \\
& =\beta_{v} \quad \mathcal{C}\left(\lambda k \cdot \operatorname{tp} J^{\bullet}[\lambda x \cdot \#(k x) / k]\right) \\
& =\quad \mathcal{C}\left(\lambda k \cdot \operatorname{tp} J[\operatorname{tp}(\lambda x . \#(k x)) \square / k]^{\bullet}\right) \\
& =\#_{l e t} \mathcal{C}\left(\lambda k \cdot \operatorname{tp} J^{\bullet}\right)
\end{array}
$$

we get:

PROPOSITION 34. For all $M$ in $\lambda_{\mathcal{C} \# t p}^{-}, M==_{\lambda_{\mathcal{C} \# t p}^{\prime}}^{\prime} M^{\bullet}$.

\footnotetext{
2 The CPS translation in (Kameyama and Hasegawa, 2003) is the composition of the Plotkin-style variants of the Fisher-style call-by-value translations $\llbracket-\rrbracket^{\prime} k$ and 【-』tp. Both styles of translation are isomorphic. By Theorem 31, they are also isomorphic to $\llbracket-k t p$ and the equations are the same.
} 
Now, take $M$ in $\lambda_{\mathcal{C} \# \text { tp }}$. We embed $M$ into $\lambda_{\mathcal{C} \# \text { tp }}^{-}$by replacing each free continuation variable $k$ in $M$ by the context tp $\left(y_{k} \square\right)$ for $y_{k}$ a fresh variable associated to $k$. Let $\rho$ be this substitution and $\rho^{\prime}$ the substitution that maps each $y_{k}$ to $\lambda x$.Th $k x$. The term $(M[\rho])^{\bullet}$ is in $\lambda_{\mathcal{C} \# \text { tp }}^{\prime}$ and, using $\mathcal{T}_{\text {let }}$, we can show that $M=_{\lambda_{\mathcal{C} \# \text { tp }}^{\prime}}^{\prime}(M[\rho])^{\bullet}\left[\rho^{\prime}\right]$.

A simple verification shows that the equation \# let used in the proof of Proposition 34 is sound with respect to $\llbracket \rrbracket k t p$. Moreover, Figure 23 shows that $\llbracket k t p$ is stable by substitution of continuation variables. Hence we get:

THEOREM 35 (Completeness). Given $\lambda_{\mathcal{C} \# \text { tp }}$ terms $M$ and $N$, from $\llbracket M \rrbracket k t p=\beta, \eta, \wedge, \eta \wedge \llbracket N \rrbracket k$ tp, we get $M={ }_{\lambda_{\mathcal{C} \# t p}^{\prime}}^{\prime} N$.

\subsection{MORE ON shift AND reset}

We now make the connection with Kameyama and Hasegawa axiomatics more precise. Let $\lambda_{\mathcal{S}}$ be the language defined by

$$
M::=x|\lambda x . M| M M|\mathcal{S}(\lambda k . M)|\langle M\rangle
$$

where $\mathcal{S}$ and \langle\rangle are primitive symbols. Clearly, passing from $\lambda_{\mathcal{C} \# \text { tp }}^{\prime}$ to $\lambda_{S}$ is just a move from macro-defined $\mathcal{S}$ and \langle\rangle to primitive $\mathcal{S}$ and \langle\rangle . We can check that all equations in $=_{\lambda_{\mathcal{C} \# \text { tp }}^{\prime}}$ not already checked valid for $=\beta, \eta, \wedge, \eta \wedge$ through the CPS translation are so. Hence, we get:

THEOREM 36 (Isomorphism between $\lambda_{\mathcal{C} \# \text { tp }}^{-}$and $\lambda_{\mathcal{S}}$ ). We have the following chain of isomorphisms:

$$
\left(\lambda_{\mathcal{C} \# t p}^{-},=_{\lambda_{\mathcal{C} \# t p}}^{\prime}\right) \simeq\left(\lambda_{\mathcal{C} \# t p}^{\prime},=_{\lambda_{\mathcal{C} \# t p}}^{\prime}\right) \simeq\left(\lambda_{\mathcal{C} \# t p}^{\prime},=_{K H}\right) \simeq\left(\lambda_{\mathcal{S}},=_{K H}\right) .
$$

Proof. The first isomorphism is by Proposition 34. The second is by Proposition 32 and by composition of CPS soundness of $={ }_{\lambda_{\mathcal{C H t p}}}$ and CPS completeness of $=K H$.

To extend the isomorphism to $\lambda_{\mathcal{C} \# \mathrm{tp}}$, that has free continuation variables, we need to extend $\lambda_{\mathcal{S}}$ with one new constant $c_{k}$ for each continuation variable $k$ in $\lambda_{\mathcal{C} \text { tp }}$. If each of this constant were constrained to occur in contexts of the form $\mathcal{S}\left(\lambda k^{\prime} . c_{k} M\right)$ or \# $(k M)$, we would directly get a correspondence with $\lambda_{\mathcal{C} \# \text { tp }}$. If we otherwise allow these constants to occur anywhere in terms, then we need to assign a default semantics (abortive or functional) to them.

Let $\lambda_{\mathcal{S}}^{+}$be the extension of $\lambda_{\mathcal{S}}$ with the constants $c_{k}$. To assign an abortive semantics, we extend $=_{K M}$ with the parametric equation 
$C_{\mathcal{A}}: c_{k}=\lambda x \cdot \mathcal{S}\left(\lambda_{-} \cdot c_{k} x\right)$. We also extend $\lambda_{\mathcal{C} \text { \#tp }}^{\prime}$ to allow free occurrences of continuation variables to the proviso that they occur in subterms of the form $\lambda x$.Th $k x$. Let $\lambda_{\mathcal{C}+\text { tp }}^{\prime+\text { be }}$ be this extension. The interpretation of $\lambda_{\mathcal{C} \# \text { tp }}^{\prime}$ as a calculus of macro-definitions for $\lambda_{\mathcal{S}}$ extends to $\lambda_{\mathcal{C} \# \text { tp }}^{++\mathcal{A}}$ and $\lambda_{\mathcal{S}}^{+}$ by setting $c_{k}=\lambda x$.Th $k x$. We extend ${ }^{\bullet}$ on $\lambda_{\mathcal{C A t p}}$ by setting $(k M)^{\bullet}=$ $(\lambda x$.Th $k x) M^{\bullet}$ for $k$ free. Because $(k M)^{\bullet}$ always occurs surrounded by tp in the translation of terms, we have $k M=\operatorname{tp}((\lambda x$. Th $k x) M)$ by $T_{\text {let }}$. Hence $M={ }_{\lambda_{\mathcal{C} \text { tp }}}^{\prime} M^{\bullet}$ still holds and $\bullet$ is an isomorphism. Expressed in $\lambda_{\mathcal{C} \# \text { tp }}^{++\mathcal{A}}, C_{\mathcal{A}}$ is derivable thanks to $\beta_{v}$ and $\mathcal{C}_{\text {idem }}$.

Alternatively, we can assign a functional semantics by replacing $\lambda x$.Th $k x$ by $\lambda x$.\# $k x$ in the constructions above. We call $\lambda_{\mathcal{C} \# \text { tp }}^{+}$the image of $\lambda_{\mathcal{S}}^{+}$where $c_{k}$ is interpreted as $\lambda x$.\# $k x$. The new equation on $\lambda_{\mathcal{S}}^{+}$is $C_{\#}: c_{k}=\lambda x$.\# $\left(c_{k} x\right)$. The equation $M={ }_{\lambda_{\mathcal{C} \# \mathrm{tp}}}^{\prime} M^{\bullet}$ holds thanks to \# $\#_{\text {let }}$ and $C_{\#}$ holds in $\lambda_{\mathcal{C} \# \text { tp }}^{++_{\#}}$ by $\beta_{v}$ and $\#_{i d e m}$.

THEOREM 37 (Isomorphism between $\lambda_{\mathcal{C} \# \text { tp }}$ and $\lambda_{\mathcal{S}}^{+}$). We have the following chain of isomorphisms:

$$
\begin{aligned}
\left(\lambda_{\mathcal{C} \# t p},=_{\lambda_{\mathcal{C} \# t p}^{\prime}}^{\prime}\right) & \simeq\left(\lambda_{\mathcal{C} \# t p}^{++_{A}},=_{\lambda_{\mathcal{C} \# t p}}^{\prime}\right) \simeq\left(\lambda_{\mathcal{C} \# t p}^{++_{A}},=_{K H+C_{\mathcal{A}}}\right) \simeq\left(\lambda_{\mathcal{S}}^{+},=_{K H+C_{\mathcal{A}}}\right) \\
& \simeq\left(\lambda_{\mathcal{C} \# t p}^{\prime+\#},=_{\lambda_{\mathcal{C} \# t p}}^{\prime}\right) \simeq\left(\lambda_{\mathcal{C} \# t p}^{\prime+\#},=_{K H+C_{\#}}\right) \simeq\left(\lambda_{\mathcal{S}}^{+},=_{K H+C_{\#}}\right)
\end{aligned}
$$

Proof. It remains to prove that from $M==_{\lambda_{\mathcal{C} \# \text { tp }}}^{\prime} N$ in $\lambda_{\mathcal{C} \# \text { tp }}^{\prime+\mathcal{A}}$, we can

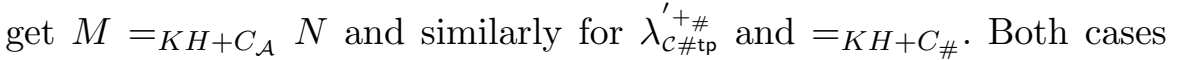
are similar and we treat only the first one. We proceed as in the proof of Theorem 35. Let $\rho$ be the substitution that maps each free continuation variables $k$ in $M$ and $N$ to tp $\left(y_{k} \square\right)$ and $\rho^{\prime}$ be the substitution that maps each $y_{k}$ to $c_{k}$ (i.e. to $\lambda x$.Th $k x$ ). From $M={ }_{\lambda_{\mathcal{C} \# \text { tp }}^{\prime}}^{\prime} N$, we get $M[\rho]={ }_{\lambda_{\mathcal{C} \# \text { tp }}}^{\prime} N[\rho]$. By composition on $\lambda_{\mathcal{C} \# \text { tp }}^{\prime}$ of CPS soundness of $={ }_{\lambda_{\mathcal{C H t p}}}$ and CPS completeness of $=_{K H}$, we get $M[\rho]={ }_{K H} N[\rho]$ and hence $M[\rho]\left[\rho^{\prime}\right]={ }_{K H} N[\rho]\left[\rho^{\prime}\right]$. It remains to connect $M[\rho]\left[\rho^{\prime}\right]$ to $M$ and $N[\rho]\left[\rho^{\prime}\right]$ to $N$. Since the free occurrences of $k$ in $M$ and $N$ occur as part of subterms of the form $\lambda x$.Th $k x$, this amounts to connect $c_{k}$ to $\lambda x$.Th tp $\left(c_{k} x\right)$. This is a direct consequence of $C_{\mathcal{A}}$ and $\beta_{v}$.

EXAMPLE 38. Let $M=\# k(f 1)+\# k(\# \operatorname{tp}(g 1))+\mathcal{T h} k(h 1)$. The image of $M$ by $\bullet$ is \# $\left(c_{k}(f 1)\right)+\#\left(c_{k} \#(g 1)\right)+\mathcal{S}\left(\lambda_{-} \cdot c_{k}(h 1)\right)$ : at this point, the interpretation of $c_{k}$ does not matter since we have tp $((\lambda x \cdot$ Th $k x) N)=T_{T_{l e t}} k N=\#_{\text {let }} \operatorname{tp}((\lambda x . \# k x) N)$.

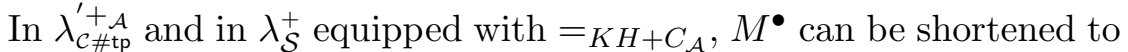
$\#\left(c_{k}(f 1)\right)+\#\left(c_{k} \#(g 1)\right)+c_{k}(h 1)$ : the abortive semantics of $\mathcal{S}\left(\lambda_{-}\right.$. $)$can be left implicit because $\mathcal{C}_{\text {tail }}$ holds. 
In $\lambda_{\mathcal{C} \# \text { tp }}^{++\#}$ and in $\lambda_{\mathcal{S}}^{+}$equipped with $={ }_{K H+C_{\#}}, M^{\bullet}$ can be shortened to $\#\left(c_{k}(f 1)\right)+c_{k} \#(g 1)+\mathcal{S}\left(\lambda_{-} . c_{k}(h 1)\right)$ : the second \# has been removed thanks to $\#_{\text {tail }}$ but the first one cannot be hidden in general.

Conversely, the expression $c_{k}(f 1)+\# c_{k}(g 1)$ in $\lambda_{\mathcal{S}}^{+}$is not explicit enough to express the semantics of the first occurrence of $c_{k}$. With an abortive semantics, it will be equivalent in $\lambda_{\mathcal{C} \# \text { tp }}$ to the expression Th $k(f 1)+\# k(g 1)$ while with a functional semantics, it will be equivalent to $(\lambda x . \# k x)(f 1)+\# k(g 1)$. Note again that $(\lambda x . \# k x)(f 1)$

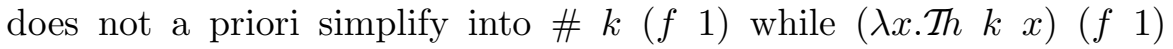
does simplify to $\mathcal{T} h k(f 1)$. Also, both \# tp $(\lambda x . \# k x)(g 1)$ and

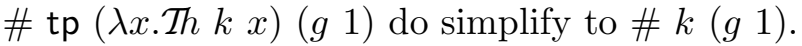

\section{Conclusions}

We have focused exclusively on delimited continuations obtained with $\mathcal{C}$ and \# (or equivalently shift and reset). We briefly review and classify some of the other control operators in the literature and discuss them based on our work. We also discuss the question of interpreting delimited continuations along the lines of the Curry-Howard correspondence.

\subsection{A SHORT History}

Early proposals for delimited continuations had only a single control delimiter (Felleisen et al., 1987; Felleisen, 1988; Danvy and Filinski, 1989). The control operation for capturing the continuation implicitly refers to the most recent occurrence of this delimiter.

After the limitations of the single control delimiter became apparent, later proposals generalised the single delimiter by allowing hierarchies of delimiters and control operators like reset ${ }_{n}$ and shift ${ }_{n}$ (Danvy and Filinski, 1990; Sitaram and Felleisen, 1990a). At about the same time, a different proposal spawn allowed new delimiters to be generated dynamically (Hieb and Dybvig, 1990; Hieb et al., 1994). In this system, the base of each delimited continuation is rooted at a different \#. The action of creating the \# returns a specialised control operator for accessing occurrences of this particular \#; this specialised control operator can then be used for capturing (and aborting) the particular delimited continuation rooted at the newly generated \# (and only that one). This is more expressive and convenient than either single delimiters or hierarchies of delimiters and allows arbitrary nesting and composition of continuation-based abstractions. A later proposal by Gunter et al. (1995) separated the operation for creating new delimiters from the control operator using that \#. 


\subsection{Control Delimiters and Extent}

The issues related to hierarchies of control delimiters or the dynamic generation of new names for control delimiters, appear orthogonal to our analysis. Indeed, the presence of multiple delimiters does not change the fundamental point about the dynamic behaviour of each individual \#.

However, our analysis fundamentally relies on a subtle issue related to the extent of delimiters (Moreau and Queinnec, 1994). More precisely, there is no question that the \# delimits the part of the context that a control operator gets to capture, but given that constraint there are still four choices to consider with very different semantics (Dybvig et al., 2004):

$$
\begin{aligned}
& E\left[\#\left(E_{\uparrow}\left[\mathcal{F}_{1} M\right]\right)\right] \mapsto E\left[M\left(\lambda x . E_{\uparrow}[x]\right)\right] \\
& E\left[\#\left(E_{\uparrow}\left[\mathcal{F}_{2} M\right]\right)\right] \mapsto E\left[\#\left(M\left(\lambda x . E_{\uparrow}[x]\right)\right)\right] \\
& E\left[\#\left(E_{\uparrow}\left[\mathcal{F}_{3} M\right]\right)\right] \mapsto E\left[M\left(\lambda x . \#\left(E_{\uparrow}[x]\right)\right)\right] \\
& E\left[\#\left(E_{\uparrow}\left[\mathcal{F}_{4} M\right]\right)\right] \mapsto E\left[\#\left(M\left(\lambda x . \#\left(E_{\uparrow}[x]\right)\right)\right)\right]
\end{aligned}
$$

All four variants have been proposed in the literature: $\mathcal{F}_{1}$ is like the operator cupto (Gunter et al., 1995); $\mathcal{F}_{2}$ is Felleisen's $\mathcal{F}$ operator (1988); $\mathcal{F}_{3}$ is like a spawn controller (Hieb and Dybvig, 1990); and $\mathcal{F}_{4}$ is shift (Danvy and Filinski, 1990).

It turns out that the inclusion of the \# in the reified continuation (variants $\mathcal{F}_{3}$ and $\mathcal{F}_{4}$ ) simplifies the semantics considerably. For example, when a continuation captured by $\mathcal{F}_{3}$ or $\mathcal{F}_{4}$ is invoked, the included \# can be used to provide the required top-level context for that invocation. In the case of $\mathcal{F}_{1}$ or $\mathcal{F}_{2}$, there is no included \#; so when the continuation is invoked, we must "search" for the \# required to denote the top-level. In general, the \# can be located arbitrarily deep in the calling context. Since the representation of contexts as delimited continuations does not support operations for "searching for delimiters," Felleisen et al. (1988) developed a special model based on an algebra of contexts which supports the required operations. In a recent investigation of control operators for delimited continuations, Dybvig et al. (2004) show however that it is possible to use standard continuation semantics to explain all the four variants of operators above: the trick is to augment the model with a state variable containing a sequence of continuations and delimiters. Chung-Chieh Shan (2004) has also recently shown a similar result using a different encoding for the operators in terms of shift and reset. We believe that these encodings can be adapted as the basis for an embedding of the operators in a variant (or extension) of $\lambda_{\mathcal{C}}^{-}$. 


\subsection{The CURRY-Howard CORRESPONDENCE}

The Curry-Howard correspondence relates proofs to programs, propositions to types, and proof normalisation to program normalisation. Typically, the simply-typed $\lambda_{\mathcal{C t p}_{\mathrm{p}}}$-calculus (alternatively Parigot's $\lambda \mu_{\mathrm{tp}}$ calculus) coincides with classical natural deduction as soon as tp is assigned the type $\perp$ (Ariola and Herbelin, 2003). Of course, this is a restriction from the point of view of any computationally interesting type system for control for which one would expect the top-level to be of an inhabited type (e.g. the type of integers). But this is really where the Curry-Howard correspondence holds, on the fragment of Figure 6 obtained by setting $T$ to $\perp$.

We mapped the effect-based type systems within subtractive logic but we did not answer the question of a direct syntactic Curry-Howardstyle interpretation.

A possible approach is the following: in the same way as the logical interpretation of tp expects it has type $\perp$, let's constrain $\widehat{t p}$ to take arguments of type $\perp$. By this way, the type system $\Lambda_{\widehat{C} \widehat{t p}}^{\rightarrow}$ fixed collapses to classical implicational logic and similarly for the type systems $\Lambda_{\widehat{\mathcal{C} p p}}^{\rightarrow}$ effeq and $\Lambda_{\widehat{\mathcal{C} t p}}^{\rightarrow}$ eff when, in addition, the effects annotations, implicitly set to $\perp$, are omitted. Along this interpretation, delimiters provide no extra logical expressiveness. This is somehow disappointing but can we really get more? After all, even if it does not enrich the logic, it still enriches the proof language. And this may well be related to completeness questions, since $\lambda \mu$-calculus is known not to satisfy Böhm theorem (David and Py, 2001) while adding delimiters is known to provide some completeness properties (Sitaram and Felleisen, 1990b; Filinski, 1994).

\section{Acknowledgements}

We thank Olivier Danvy, Matthias Felleisen, Yukiyoshi Kameyama, and Hayo Thielecke for the discussions and help they provided in understanding their results. We would also like to thank the ICFP and HOSC reviewers who provided corrections and extensive comments on the presentation of both the conference and journal versions. 


\section{Appendix}

\section{A. Proof of strong normalisation of $\lambda_{\mathcal{C}}^{-}$}

The $\lambda_{\mathcal{C}}^{-}$calculus is defined by the reduction rules in Figure 18. We show that its simply-typed fragment, as described in Figure 19, is strongly normalisable (SN).

We begin by establishing a closure property of SN that is needed in the rest of the proof. We then generalise the notion of SN to that of reducibility and show that all typed terms are reducible.

In the following we say that $M$ (respectively $E$ or $J_{c}[E]$ ) is SN if all its immediate reducts are. This is an inductive definition so that we can reason by structural induction on it.

\section{A.1. Properties of SN}

As the following establishes, strong normalisability is preserved by head expansion.

LEMMA 39.

1. If $V$ and $J_{c}[E[M[V / x]]]$ are $S N$, then $J_{c}[E[(\lambda x . M) V]]$ is $S N$

2. If $J_{c}[E], V$ and $J[V / y]\left[J_{c}[E] / k\right]$ are $S N$ then we also have that:

let $(y, k)=\left(V, \lambda x . J_{c}[E[x]]\right)$ in $J$ is $S N$

3. If $J_{c}[E]$ and $J\left[J_{c}[E] / k\right]$ are $S N$ then $J_{c}[E[\mathcal{C}(\lambda k . J)]]$ is $S N$.

Proof.

1. We reason by induction on the SN proofs. If a reduction step occurs in $J_{c}[E]$ leading to $J_{c}^{\prime}\left[E^{\prime}\right]$ then $J_{c}[E[M[V / x]]] \rightarrow J_{c}^{\prime}\left[E^{\prime}[M[V / x]]\right]$ and the induction hypothesis on $J_{c}^{\prime}\left[E^{\prime}[M[V / x]]\right]$ SN applies. Similarly for a reduction step in $M$. If a reduction step occurs in $V$ leading to $V^{\prime}$ then $J_{c}[E[M[V / x]]] \rightarrow J_{c}\left[E\left[M\left[V^{\prime} / x\right]\right]\right]$. Hence, the jump $J_{c}\left[E\left[M\left[V^{\prime} / x\right]\right]\right]$ is $\mathrm{SN}$ and the induction hypothesis on $V^{\prime} \mathrm{SN}$ applies. Finally, if $J_{c}[E[(\lambda x \cdot M) V]] \rightarrow J_{c}[E[M[V / x]]]$, the result is $\mathrm{SN}$ by hypothesis.

2. Let $\left.J^{\prime}=J[V / x]\left[J_{c}[E] / k\right]\right]$. We reason by induction on the structure of $E$, then by induction on the proofs of SN for $J_{c}[E], V$, and $J^{\prime}$. If $E=\square$, then a reduction step in let $(x, k)=\left(V, \lambda x . J_{c}[x]\right)$ in $J$ occurs either in $J_{c}, J, V$ or it yields $J^{\prime}$. In the first cases, we apply the induction hypothesis as above. In the latter case, the 
contractum is directly SN by hypothesis. Otherwise, if $E$ is some $E^{\prime}[F]$, we consider the different reduction steps that can occur in let $(x, k)=\left(V, \lambda x . J_{c}\left[E^{\prime}[F[x]]\right]\right)$ in $J$ :

- If the reduction step occurs in $V$ (respectively $J_{c}\left[E^{\prime}[F]\right]$ ) leading to $V^{\prime}$ (respectively $J_{c}^{\prime \prime}\left[E^{\prime \prime}\right]$ ), then $\left.J^{\prime} \rightarrow J\left[V^{\prime} / x\right]\left[J_{c}[E] / k\right]\right]$ (respectively $\left.J^{\prime} \rightarrow J[V / x]\left[J_{c}^{\prime \prime}\left[E^{\prime \prime}\right] / k\right]\right]$ ) so that the reducts of $J^{\prime}$ and $V$ (respectively $J_{c}\left[E^{\prime}[F]\right]$ ) are $\mathrm{SN}$ and the induction hypothesis applies.

- If the reduction step occurs in $J$ leading to some jump $J^{\prime \prime}$, then $\left.J^{\prime} \rightarrow J^{\prime \prime}[V / x]\left[J_{c}[E] / k\right]\right]$ so that the latter reduct is SN and the induction hypothesis applies.

- If the reduction gives:

let $(x, k)=\left(V, \lambda x . J_{c}\left[E^{\prime}[x]\right]\right)$ in $J[k F / k]$ then $J^{\prime}$ can be rewritten to $J[k F / k][V / x]\left[J_{c}\left[E^{\prime}\right] / k\right]$ so that the induction hypothesis on $E$ applies (taking $E^{\prime}$ and $J[k F / k]$ for $E$ and $J$ respectively)

3. As above, we reason by induction on the structure of $J_{c}[E]$, then by induction on the proofs of SN for $J_{c}[E]$, and $J\left[J_{c}[E] / k\right]$. If $E=\square$, then there are two interesting cases:

- If $J_{c}[\mathcal{C}(\lambda k . J)] \rightarrow J\left[J_{c} / k\right]$ (i.e. $\mathcal{C}_{\text {idem }}$ or $S u b_{v}^{\text {lift }}$ ), then SN follows from the hypothesis.

- If $J$ is some $k M$ with $k$ not free in $M$ and $J_{c}[\mathcal{C} k . J] \rightarrow J_{c}[M]$ using $\mathcal{C}_{\text {elim }}$, then the reasoning is the same since $J_{c}[M]=$ $J\left[J_{c} / k\right]$.

If $E$ is some $E^{\prime}[F]$, then there are also two interesting cases:

- If $J_{c}\left[E^{\prime}[F[\mathcal{C}(\lambda k . J)]]\right] \rightarrow J_{c}\left[E^{\prime}[\mathcal{C}(\lambda k . J[k F / k])]\right]$, then SN follows by induction hypothesis on $E^{\prime}$ since $J\left[J_{c}\left[E^{\prime}[F]\right] / k\right]$ can be rewritten into $J[k F / k]\left[J_{c}\left[E^{\prime}\right] / k\right]$.

- If $J$ is some $k M$ with $k$ not free in $M$ and $J_{c}[E[\mathcal{C} k . J]] \rightarrow$ $J_{c}[E[M]]$ using $\mathcal{C}_{\text {elim }}$, then again, the reasoning is the same since $J_{c}[E[M]]=J\left[J_{c}[E] / k\right]$.

\section{A.2. Reducibility}

We define the property "reducible of a given type" for terms and jump contexts. The definition is by induction on the type, then by mutual induction on values, jump contexts, and non-value terms, with priority given first to values, then to jump contexts, and finally to non-values. 
The definition is a straightforward adaptation of the notion of reducibility developed by Herbelin (2001) for proving strong normalisation of the call-by-name $\bar{\lambda} \mu \tilde{\mu}$-calculus.

DEFINITION 40. [Reducible of a given type]

- A value $V$ is reducible of type $A$ iff:

- $V$ is a variable

- $V$ is $\lambda x . N$ and $A$ is some $B \rightarrow C$ such that for all value $V^{\prime}$ reducible of type $B, V V^{\prime}$ is reducible of type $C$

- $\quad V$ is $\left(V^{\prime}, \lambda x . J_{c}[E[x]]\right)$ and $A$ is some $B-C$ and $V^{\prime}$ is reducible of type $B$ and $J_{c}[E]$ is reducible of type $C$.

- A jump context $J_{c}[E]$ is reducible of type $A$ iff for all value $V$ reducible of type $A, J_{c}[E[V]]$ is $\mathrm{SN}$.

- A non-value term $M$ is reducible of type $A$ iff for all jump contexts $J_{c}[E]$ reducible of type $A, J_{c}[E[M]]$ is $\mathrm{SN}$.

Remark 1. Since reducible jump contexts are SN against any reducible value, the if part of the last clause of the definition of reducibility also holds for values.

\section{A.3. Properties of Reducibility}

LEMMA 41. Reducible values, non-value terms and contexts are $S N$ :

1. For all $J_{c}[E]$ reducible of type $A, J_{c}[E]$ is $S N$.

2. For all $M$ reducible of type $A, M$ is $S N$.

3. For all $k$ of type $A, k \square$ is a reducible jump context.

Proof. Let $J_{c}[E]$ be a reducible jump context. Take a variable $x$. It is a reducible value, hence $J_{c}[E[x]]$ is SN. Especially $J_{c}[E]$ is $\mathrm{SN}$.

We show the last two items conjointly by induction on $A$. We first show that $M$ reducible is $\mathrm{SN}$ if it is a value.

- If $M$ is a variable, it is $\mathrm{SN}$.

- If $M$ is $\lambda x . N$ of type $B \rightarrow C$, then applying it to $x$ (which is reducible of type $B$ ) yields a reducible term of type $C$ identical to $N$. By induction hypothesis on $C, N$ is $\mathrm{SN}$ so that $M$ is $\mathrm{SN}$ too. 
- If $M$ is $(V, k E)$ of type $B-C$ with $k E$ reducible of type $C$ and $V$ reducible of type $B$ then $k E$ is $\mathrm{SN}$, and $V$ is $\mathrm{SN}$ by induction hypothesis, so that $M$ is $\mathrm{SN}$.

We then show that all jump contexts of the form $k \square$ are reducible of type $A$. Let $V$ be a reducible value of type $A$. We have to show that $k V$ is SN. We just showed that $V$ was SN. Since the only redexes of $k V$ are redexes of $V$, we get $k V \mathrm{SN}$.

We can now extend the result to any non-value $M$. Let $k$ be a continuation variable of type $A$. Since $k \square$ is reducible of type $A$, we obtain that $k M$ is $\mathrm{SN}$. Especially, $M$ is $\mathrm{SN}$.

The following lemma shows that reducibility of jump contexts is preserved by context construction.

LEMMA 42. We have:

1. If $V$ is reducible of type $A \rightarrow B$ and $J_{c}[E]$ reducible of type $B$ then $J_{c}[E[V \square]]$ is reducible of type $A$.

2. If $M$ is reducible of type $A$ and $J_{c}[E]$ reducible of type $B$ then $J_{c}[E[\square M]]$ is reducible of type $A \rightarrow B$.

3. If $J_{c}[E]^{\prime}$ is reducible of type $A$ and $J_{c}[E]$ reducible of type $B-A$ then $J_{c}\left[E\left[\left(\square, \lambda x . J_{c}^{\prime}\left[E^{\prime}[x]\right]\right)\right]\right]$ is reducible of type $B$.

4. If $J[V / x]\left[J_{c}[E] / k^{\prime}\right]$ is $S N$ for any reducible $V$ of type $B$ and $J_{c}[E]$ of type $C$ then let $\left(x, k^{\prime}\right)=\square$ in $J$ is a reducible jump context of type $B-C$.

Proof.

1. We have to show that $J_{c}\left[E\left[V V^{\prime}\right]\right]$ is $\mathrm{SN}$ for any $V^{\prime}$ reducible of type $A$. If $V$ is some variable $x$ then redexes of $J_{c}\left[E\left[x V^{\prime}\right]\right]$ are either in $J_{c}[E]$ or in $V^{\prime}$ which are SN by Lemma 2. If $V$ is some $\lambda x . M$ then, by definition of its reducibility, $V V^{\prime}$ is reducible. Combined with the reducibility of $J_{c}[E]$, we get $J_{c}\left[E\left[V V^{\prime}\right]\right] \mathrm{SN}$.

2. We have to show that $J_{c}[E[V M]]$ is SN for any $V$ reducible of type $A \rightarrow B$. By the previous item, $J_{c}[E[V \square]]$ is a reducible context of type $A$. Hence, $J_{c}[E[V M]]$ is $\mathrm{SN}$, by reducibility of $M$.

3. We have to show that $J_{c}\left[E\left[\left(V, \lambda x . J_{c}^{\prime}\left[E^{\prime}[x]\right]\right)\right]\right]$ is SN for any $V$ reducible of type $B$. The reducibility of $J_{c}^{\prime}\left[E^{\prime}\right]$ and $V$ implies the reducibility of the value $\left(V, \lambda x . J_{c}^{\prime}\left[E^{\prime}[x]\right]\right)$. Hence, the reducibility of $J_{c}[E]$ implies $J_{c}\left[E\left[\left(V, \lambda x . J_{c}^{\prime}\left[E^{\prime}[x]\right]\right)\right]\right] \mathrm{SN}$. 
4. We have to show that let $\left(x, k^{\prime}\right)=V$ in $J$ is $\mathrm{SN}$ for any $V$ reducible of type $B-C$. If $V$ is some variable $y$ then the only redexes of let $\left(x, k^{\prime}\right)=y$ in $J$ are in $J$. Since $J=J[x / x]\left[k^{\prime} \square / k^{\prime}\right]$ which is SN by hypothesis, the whole expression is SN too. If $V$ is some value of the form $\left(V^{\prime}, \lambda x . J_{c}^{\prime \prime}\left[E^{\prime \prime}[x]\right]\right)$, then by hypothesis, $J\left[V^{\prime} / x\right]\left[J_{c}^{\prime \prime}\left[E^{\prime \prime}\right] / k^{\prime}\right]$ is SN. By Lemma 39(2), we conclude that let $\left(x, k^{\prime}\right)=\left(V^{\prime}, J_{c}^{\prime \prime}\left[E^{\prime \prime}\right]\right)$ in $J$ is $\mathrm{SN}$ too.

\section{A.4. Adequacy Lemma}

Finally, we show the main lemma that all typed terms are reducible.

LEMMA 43. Let $\Gamma$ be an ordered context of declarations of the form either $x_{i}: A_{i}$ or $k_{i}: B_{i} \rightarrow \Perp$. Let $V_{i}$ be instances for the variables $x_{i}$ and $J_{c}^{i}\left[E_{i}\right]$ be instances for the variables $k_{i}$ such that the free variables of $V_{i}$ and $J_{c}^{i}\left[E_{i}\right]$ are among the $x_{j}$ and $k_{j}$ for $j \leq i$. The $V_{i}$ are reducible values of respective types $A_{i}$ and the $J_{c}^{i}\left[E_{i}\right]$ are reducible jump contexts of respective types $B_{i}$. We write $[\sigma]$ for the ordered substitution mixing the substitution $\left[V_{i} / x_{i}\right]$ and $\left[J_{c}^{i}\left[E_{i}\right] / k_{i}\right]$. We have:

- $, \Delta \vdash M: A$ implies $M[\sigma]$ reducible of type $A$

$-\Gamma, \Delta \vdash J: \Perp$ implies $J[\sigma] S N$.

Proof. We reason by induction on the derivation of $\Gamma, \Delta \vdash M: A$ or $\Gamma, \Delta \vdash J: \Perp$.

- Rule $A x$ with $M=x_{i}$ : this is direct by reducibility of $V_{i}$.

- Rule $\rightarrow e$ with $M=M_{1} M_{2}$ with $M_{1}$ of type $B \rightarrow A$ and $M_{2}$ of type $B$. Let $J_{c}[E]$ a reducible jump context of type $A$. Since $M_{2}^{\prime}=M_{2}[\sigma]$ is reducible of type $B$ by induction hypothesis, $J_{c}\left[E\left[\square M_{2}^{\prime}\right]\right]$ is reducible of type $B \rightarrow A$ by Lemma $42(2)$. Since $M_{1}^{\prime}=M_{1}[\sigma]$ is reducible by induction hypothesis, we have that $J_{c}\left[E\left[M_{1}^{\prime} M_{2}^{\prime}\right]\right]$ is SN. Hence $M[\sigma]=M_{1}^{\prime} M_{2}^{\prime}$ is reducible.

- Rule $\rightarrow_{i}$ with $M=\lambda x . N: A \rightarrow B$. Let $V$ be a reducible value of type $A$ and $J_{c}[E]$ a reducible jump context of type $B$. By induction hypothesis, we have $N[\sigma][V / x]$ reducible of type $B$ hence $J_{c}[E[N[\sigma][V / x]]]$ is SN. By Lemma $41, V$ is SN. By Lemma 39(1), we get $J_{c}[E[(\lambda x .(N[\sigma])) V]] \mathrm{SN}$ so that $(\lambda x . N)[\sigma]=\lambda x .(N[\sigma])$ is reducible. 
- Rule $\rightarrow{ }_{e}^{k}$ with $J=k_{i_{0}} M$ and $M$ of type $A$ and $k_{i_{0}}$ of type $A \rightarrow \Perp$. By induction hypothesis we have $M^{\prime}=M[\sigma]$ reducible of type $A$. Since $J_{c}^{i_{0}}\left[E_{i_{0}}\right]$ is reducible (of type A), we get $J[\sigma]=J_{c}^{i_{0}}\left[E_{i_{0}}\right]\left[M^{\prime}\right]$ $\mathrm{SN}$.

- Rule $R A A$ with $M=\mathcal{C}(\lambda k . J)$. Let $J_{c}[E]$ a reducible jump context of type $A$. By induction hypothesis we have $J^{\prime}=J[\sigma]\left[J_{c}[E] / k\right] \mathrm{SN}$. By Lemma $41, J_{c}[E]$ is SN so that we get $J_{c}[E[\mathcal{C}(\lambda k . J)]]$ SN by Lemma 39(3).

- Rule $-_{e}$ with $J_{0}=($ let $(y, k)=N$ in $J)$ with $N$ of type $B-C$. We have to show that $J_{0}[\sigma]$ is SN. By induction hypothesis we already know that $N^{\prime}=N[\sigma]$ is reducible of type $B-C$. Let $J^{\prime}=J[\sigma]$. By induction hypothesis, we have that $J^{\prime}[V / y]\left[J_{c}[E] / k\right]$ is SN for every reducible $V$ of type $B$ and $J_{c}[E]$ of type $C$. Hence, by Lemma 42(4), let $(y, k)=\square$ in $J^{\prime}$ is a reducible jump context of type $B-C$. By reducibility of $N^{\prime}$, we conclude that $J_{0}[\sigma]=$ let $(y, k)=N^{\prime}$ in $J^{\prime}$ is $\mathrm{SN}$.

- Rule $-i$ with $M=\left(M, \lambda x . J_{c}[E[x]]\right)$ and $A=B-C$. The typability of $J_{c}[E]$ states that $\Gamma, \Delta, x: C \vdash J_{c}[E[x]]: \Perp$ for some fresh variable $x$. By induction hypothesis, $\left(\left(J_{c}[E]\right)[\sigma]\right)[V]=J_{c}[E[x]][\sigma][V / x]$ is SN for any reducible $V$ of type $C$ so that $J_{c}^{\prime}\left[E^{\prime}\right]=\left(J_{c}[E]\right)[\sigma]$ is reducible of type $C$. By induction hypothesis, $N^{\prime}=N[\sigma]$ is reducible too, of type $B$. If $N^{\prime}$ is a value, we get that $M[\sigma]=$ $\left(N^{\prime}, \lambda x . J_{c}^{\prime}\left[E^{\prime}[x]\right]\right)$ is reducible. Otherwise, we have to show that $J_{c}^{\prime \prime}\left[E^{\prime \prime}\left[\left(N^{\prime}, \lambda x . J_{c}^{\prime}\left[E^{\prime}[x]\right]\right)\right]\right]$ is SN for every reducible jump context $J_{c}^{\prime \prime}\left[E^{\prime \prime}\right]$ of type $B-C$. By Lemma $42(3), J_{c}^{\prime \prime}\left[E^{\prime \prime}\left[\left(\square, \lambda x . J_{c}^{\prime}\left[E^{\prime}[x]\right]\right)\right]\right]$ is reducible of type $B$. Hence, by reducibility of $N^{\prime}$, we conclude that $J_{c}^{\prime \prime}\left[E^{\prime \prime}\left[\left(N^{\prime}, \lambda x . J_{c}^{\prime}\left[E^{\prime}[x]\right]\right)\right]\right]$ is $\mathrm{SN}$.

Combining the above with Lemma 41(1 and 2), and because variables and variable-based jump contexts are reducible (respectively by definition and by Lemma 41(3)), we finally get the strong normalisability of $\lambda_{\mathcal{c}}^{-}$.

THEOREM 44. Typed $\lambda_{\mathcal{C}}^{-}$is strongly normalisable.

\section{References}

Ariola, Z. M. and H. Herbelin: 2003, 'Minimal Classical Logic and Control Operators'. In: Thirtieth International Colloquium on Automata, Languages and Programming , ICALP'03, Eindhoven, The Netherlands, June 30 - July 4, 2003, Vol. 2719. pp. 871-885, Springer-Verlag, LNCS. 
Ariola, Z. M. and H. Herbelin: 2007, 'Control Reduction Theories: The Benefit of Structural Substitution'. Journal of Functional Programming. To appear.

Ariola, Z. M., H. Herbelin, and A. Sabry: 2004, 'A Type-Theoretic Foundation of Continuations and Prompts'. In: ACM SIGPLAN International Conference on Functional Programming. pp. 40-53, ACM Press, New York.

Baba, K., S. Hirokawa, and K. etsu Fujita: 2001, 'Parallel Reduction in Type Free $\lambda \mu$-Calculus'. Electronic Notes in Theoretical Computer Science 42, 52-66.

Barbanera, F. and S. Berardi: 1993, 'Extracting Constructive Content from Classical Logic via Control-Like Reductions'. In: M. Bezem and J. F. Groote (eds.): Proceedings 1st Intl. Conf. on Typed Lambda Calculi and Applications, TLCA'93, Utrecht, The Netherlands, 16-18 March 1993, Vol. 664. Berlin: Springer-Verlag, pp. $45-59$.

Crolard, T.: 2001, 'Subtractive logic'. Theor. Comput. Sci. 254(1-2), 151-185.

Crolard, T.: 2004, 'A formulae-as-types interpretation of subtractive logic'. Journal of Logic and Computation (Special issue on Modalities in Constructive Logics and Type Theories) 14(4), 529-570.

Curien, P.-L. and H. Herbelin: 2000, 'The duality of computation'. In: ACM SIGPLAN International Conference on Functional Programming. pp. 233-243, ACM Press, New York.

Danvy, O. and A. Filinski: 1989, 'A Functional Abstraction of Typed Contexts'. Technical Report 89/12, DIKU, University of Copenhagen, Copenhagen, Denmark.

Danvy, O. and A. Filinski: 1990, 'Abstracting Control'. In: Proceedings of the 1990 ACM Conference on LISP and Functional Programming, Nice. pp. 151-160, ACM Press, New York.

David, R. and W. Py: 2001, 'Lambda-mu-Calculus and Böhm's Theorem'. Journal of Symbolic Logic 66(1), 407-413.

de Groote, P.: 1994, 'On the Relation between the lambda-mu Calculus and the Syntactic Theory of Sequential Control'. In: F. Pfennig (ed.): Logic Programming and Automated Reasoning, Proc. of the 5th International Conference, LPAR'94. Berlin, Heidelberg: Springer, pp. 31-43.

Dybvig, R. K., S. Peyton-Jones, and A. Sabry: 2004, 'A Monadic Framework for Subcontinuations'. Submitted for publication.

Felleisen, M.: 1988, 'The Theory and Practice of First-Class Prompts'. In: Proceedings of the 15th ACM Symposium on Principles of Programming Languages (POPL '88). pp. 180-190, ACM Press, New York.

Felleisen, M.: 1990, 'On the Expressive Power of Programming Languages'. In: N. Jones (ed.): ESOP '90 3rd European Symposium on Programming, Copenhagen, Denmark, Vol. 432. New York, N.Y.: Springer-Verlag, pp. 134-151.

Felleisen, M., D. Friedman, and E. Kohlbecker: 1987, 'A syntactic theory of sequential control'. Theoretical Computer Science 52(3), 205-237.

Felleisen, M. and R. Hieb: 1992, 'The Revised Report on the Syntactic Theories of Sequential Control and State'. Theoretical Computer Science 103(2), 235-271.

Felleisen, M., M. Wand, D. P. Friedman, and B. F. Duba: 1988, 'Abstract continuations: A mathematical semantics for handling full functional jumps'. In: In Conference on LISP and Functional Programming, Snowbird, Utah. pp. 52-62, ACM Press, New York.

Filinski, A.: 1989, 'Declarative Continuations: an Investigation of Duality in Programming Language Semantics'. In: Category Theory and Computer Science, Manchester, UK, September 5-8, 1989, Proceedings, Vol. 389 of LNCS. pp. 224-249, Springer-Verlag. 
Filinski, A.: 1994, 'Representing Monads'. In: Conf. Record 21st ACM SIGPLANSIGACT Symp. on Principles of Programming Languages, POPL'94, Portland, OR, USA, 17-21 Jan. 1994. pp. 446-457, ACM Press, New York.

Filinski, A.: 1999, 'Representing layered monads'. In: Proceedings of the 26th ACM SIGPLAN-SIGACT symposium on Principles of programming languages. pp. 175-188, ACM Press, New York.

Fischer, M. J.: 1972, 'Lambda-calculus schemata'. In: Proc. ACM Conference on Proving Assertions About Programs, Vol. 7(1) of SIGPLAN Notices. pp. 104-109, ACM Press, New York.

Fischer, M. J.: 1993, 'Lambda-Calculus Schemata'. LISP and Symbolic Computation 6(3/4), 259-288. <http://www.brics.dk/ hosc/vol06/03-fischer.html> Earlier version available in the proceedings of an ACM Conference on Proving Assertions about Programs, SIGPLAN Notices, Vol. 7, No. 1, January 1972.

Griffin, T. G.: 1990, 'The Formulae-as-Types Notion of Control'. In: Conf. Record 17th Annual ACM Symp. on Principles of Programming Languages, POPL'90, $S$ an Francisco, CA, USA, 17-19 Jan 1990. pp. 47-57, ACM Press, New York.

Gunter, C. A., D. Rémy, and J. G. Riecke: 1995, 'A Generalization of Exceptions and Control in ML-like Languages'. In: Functional Programming $\&$ Computer Architecture. New York, ACM Press.

Guzmán, J. and A. Suárez: 1994, 'An Extended Type System for Exceptions'. In: Record of the fifth ACM SIGPLAN workshop on ML and its Applications. Also appeared as Research Report 2265, INRIA, BP 105 - 78153 Le Chesnay Cedex, France.

Haynes, C. T.: 1986, 'Logic Continuations'. In: Proceedings of the Third International Conference on Logic Programming, Vol. 225 of Lecture Notes in Computer Science. Berlin, pp. 671-685, Springer-Verlag.

Haynes, C. T., D. Friedman, and M. Wand: 1986, 'Obtaining coroutines from continuations'. Journal of Computer Languages 11, 143-153.

Herbelin, H.: 2001, 'Explicit Substitutions and Reducibility'. Journal of Logic and Computation 11(3), 431-451.

Hieb, R., K. Dybvig, and C. W. Anderson, III: 1994, 'Subcontinuations'. Lisp and Symbolic Computation 7(1), 83-110.

Hieb, R. and R. K. Dybvig: 1990, 'Continuations and Concurrency'. In: PPoPP '90, Symposium on Principles and Practice of Parallel Programming, Vol. 25(3) of SIGPLAN NOTICES. Seattle, Washington, March 14-16, pp. 128-136, ACM Press, New York.

Hofmann, M.: 1995, 'Sound and complete axiomatisations of call-by-balue control operators'. Mathematical Structures in Computer Science 5(4), 461-482.

Howard, W.: 1980, 'The formulae-as-types notion of construction'. In: J. R. Hindley and J. P. Seldin (eds.): To H. B. Curry: Essays in Combinatory Logic, Lambda Calculus and Formalism. Academic Press, pp. 479-490.

Kameyama, Y.: 2000, 'A Type-Theoretic Study on Partial Continuations'. In: IFIP TCS. pp. 489-504.

Kameyama, Y.: 2001, 'Towards Logical Understanding of Delimited Continuations'. In: Proceedings of the Third ACM SIGPLAN Workshop on Continuations (CW'01).

Kameyama, Y. and M. Hasegawa: 2003, 'A Sound and Complete Axiomatization of Delimited Continuations'. In: Proc. of 8th ACM SIGPLAN Int. Conf. on Functional Programming, ICFP'03, Uppsala, Sweden, 25-29 Aug. 2003, Vol. 38(9) of SIGPLAN Notices. ACM Press, New York, pp. 177-188. 
Lillibridge, M.: 1999, 'Unchecked Exceptions Can be Strictly More Powerful Than Call/CC'. Higher-Order and Symbolic Computation 12(1), 75-104.

Moggi, E.: 1989, 'Computational lambda-calculus and monads'. In: Proceedings of the Fourth Annual Symposium on Logic in computer science. pp. 14-23, IEEE Press.

Moreau, L.: 1998, 'A Syntactic Theory of Dynamic Binding'. Higher Order Symbol. Comput. 11(3), 233-279.

Moreau, L. and C. Queinnec: 1994, 'Partial Continuations as the Difference of Continuations. A Duumvirate of Control Operators'. In: International Conference on Programming Language Implementation and Logic Programming (PLILP'94). Madrid, Spain, pp. 182-197, Springer-Verlag.

Murthy, C.: 1992, 'Control Operators, Hierarchies, and Pseudo-Classical Type Systems: A-Translation at Work'. In: ACM workshop on Continuations. pp. 49-71.

Ong, C.-H. L. and C. A. Stewart: 1997, 'A Curry-Howard Foundation for Functional Computation with Control'. In: Conf. Record 24th ACM SIGPLAN-SIGACT Symp. on Principles of Programming Languages, POPL'97, Paris, France, 15-17 Jan. 1997. ACM Press, New York, pp. 215-227.

Parigot, M.: 1992, 'Lambda-mu-calculus: An algorithmic interpretation of classical natural deduction'. In: Logic Programming and Automated Reasoning: International Conference LPAR '92 Proceedings, St. Petersburg, Russia. pp. 190-201, Springer-Verlag.

Rauszer, C.: 1974, 'Semi-boolean algebras and their application to intuitionistic logic with dual connectives'. Fundamenta Mathematicae 83, 219-249.

Riecke, J. G. and H. Thielecke: 1999, 'Typed Exceptions and Continuations Cannot Macro-Express Each Other'. In: Proceedings of the 26th International Colloquium on Automata, Languages and Programming (ICALP), Vol. 1644 of Lecture Notes in Computer Science. Berlin, pp. 635-644, Springer-Verlag.

Shan, C.: 2004, 'Shift to Control'. In: O. Shivers and O. Waddell (eds.): Proceedings of the 5th workshop on Scheme and Functional Programming. pp. 99-107. Technical report, Computer Science Department, Indiana University, 2004.

Sitaram, D. and M. Felleisen: 1990a, 'Control delimiters and their hierarchies'. Lisp and Symbolic Computation 3(1), 67-99.

Sitaram, D. and M. Felleisen: 1990b, 'Reasoning with continuations II: full abstraction for models of control'. In: LFP '90: Proceedings of the 1990 ACM conference on LISP and functional programming. pp. 161-175, ACM Press.

Thielecke, H.: 2000, 'On Exceptions versus Continuations in the Presence of State'. In: Proceedings of the ninth European Symposium On Programming (ESOP), Vol. 1782 of Lecture Notes in Computer Science. Berlin, pp. 397-411, Springer-Verlag.

Thielecke, H.: 2001, 'Contrasting Exceptions and Continuations'. Available from http://www.cs.bham.ac.uk/ hxt/research/exncontjournal.pdf.

Thielecke, H.: 2002, 'Comparing Control Constructs by Double-barrelled CPS'. Higher-order and Symbolic Computation 15(2/3), 119-136.

Wadler, P.: 1994, 'Monads and composable continuations.'. Lisp and Symbolic Computation $\mathbf{7}(1), 39-56$.

Wand, M.: 1999, 'Continuation-based multiprocessing'. Higher-Order and Symbolic Computation 12(3), 285-299. <http://www.brics.dk/ ^hosc/vol12/3-wand. html> Reprinted from the proceedings of the 1980 Lisp Conference, with a foreword. 
final.tex; $11 / 04 / 2007 ; 15: 30 ;$ p.56 